\title{
First-principles calculations of the Berry curvature of Bloch states for charge and spin transport of electrons
}

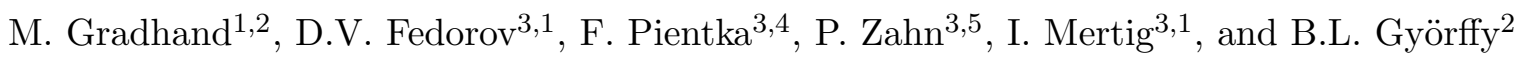 \\ 1 - Max-Planck-Institut für Mikrostrukturphysik, Weinberg 2, D-06120 Halle, Germany \\ 2 - H.H.Wills Physics Laboratory, University of Bristol, Bristol BS8 1TH, United Kingdom \\ 3 - Institut für Physik, Martin-Luther-Universität Halle-Wittenberg, D-06099 Halle, Germany \\ 4 - Dahlem Center for Complex Quantum Systems and Fachbereich Physik, Freie Universität \\ Berlin, 14195 Berlin, Germany \\ 5 - Helmholtz-Zentrum Dresden-Rossendorf e.V., P.O. Box 510119, D-01314 Dresden
}

\begin{abstract}
Recent progress in wave packet dynamics based on the insight of M. V. Berry pertaining to adiabatic evolution of quantum systems has led to the need that a new property of a Bloch state, the Berry curvature, has to be calculated from first principles. We report here on the response to this challenge by the $a b$ initio community during the past decade. First we give a tutorial introduction of the conceptual developments we mentioned above. Then we describe four methodologies which have been developed for first-principles calculations of the Berry curvature. Finally, to illustrate the significance of the new developments, we report some results of calculations of interesting physical properties like the anomalous and spin Hall conductivity as well as the anomalous Nernst conductivity and discuss the influence of the Berry curvature on the de Haas-van Alphen oscillation.
\end{abstract}

\section{Introduction: Semiclassical electronic transport in solids, wave packet dynamics, and the Berry curvature}

Some recent, remarkable advances in the semiclassical description of charge and spin transport by electrons in solids motivate a revival of interest in a detailed $\mathbf{k}$-point by $\mathbf{k}$-point study of their band structure. A good summary of the conceptual developments and the experiments they have stimulated has been given by Di Xiao, Ming-Che Chang and Qian Niu [1]. Here we wish to review only the role and prospects of first-principles electronic structure calculations in this rapidly moving field.

\subsection{New features of the Boltzmann equation}

Classically, particle transport is considered to take place in phase space whose points are labeled by the position vector $\mathbf{r}$ and the momentum $\mathbf{p}$ and described by the distribution function $f(\mathbf{r}, \mathbf{p}, \mathrm{t})$ which satisfies the Boltzmann equation. Electrons in solids fit into this framework if we view the particles as wave packets constructed from the Bloch waves, $\Psi_{n \mathbf{k}}(\mathbf{r})=e^{i \mathbf{k} \cdot \mathbf{r}} u_{n}(\mathbf{r}, \mathbf{k})$, eigenfunctions of the unperturbed crystal Hamiltonian $\hat{H}_{0}$ with band index $n$ and the dispersion relation $\mathcal{E}_{n \mathbf{k}}$. If such a wave packet is strongly centered at $\mathbf{k}_{c}$ in the Brillouin zone, it predominantly includes Bloch states with quantum numbers $\mathbf{k}$ close to $\mathbf{k}_{c}$ and the particle can be said to have a momentum $\hbar \mathbf{k}_{c}$. Then, if we call its spatial center $\mathbf{r}_{c}$ its position, the transport of 

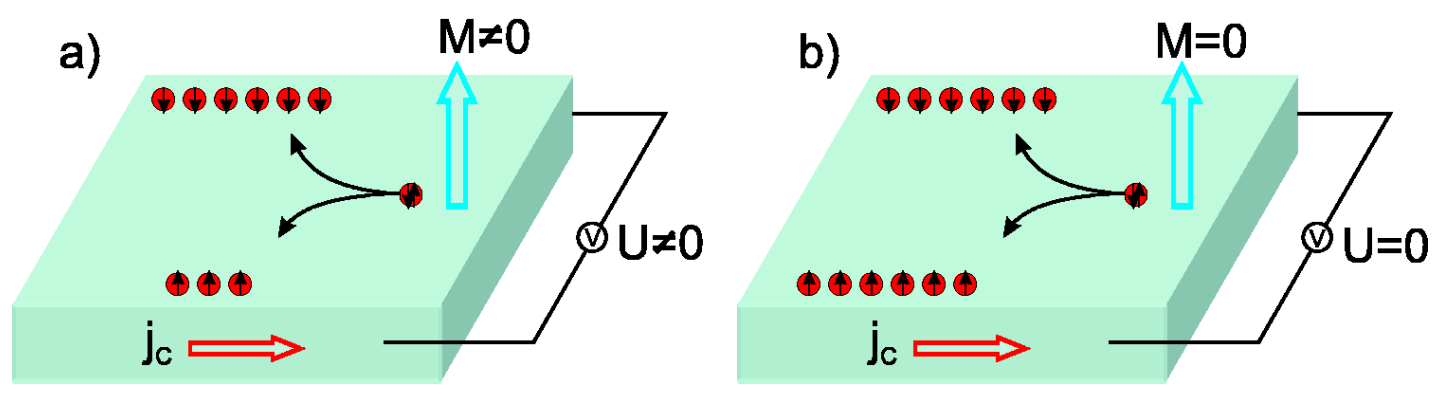

Figure 1: a) The anomalous Hall effect and b) the spin Hall effect.

electrons can be described by an ensemble of such quasiparticles. One of them is described by the distribution function $f_{n}\left(\mathbf{r}_{c}^{n}, \mathbf{k}_{c}^{n}, \mathrm{t}\right)$. For simplicity the band index $n$ is dropped in the following for the distribution function $f, \mathbf{r}_{c}$ and $\mathbf{k}_{c}$. As $f\left(\mathbf{r}_{c}, \mathbf{k}_{c}, \mathrm{t}\right)$ evolves in phase space the usual form of the Boltzmann equation, which preserves the total probability, is given by

$$
\partial_{t} f+\dot{\mathbf{r}}_{c} \cdot \nabla_{\mathbf{r}_{c}} f+\dot{\mathbf{k}}_{c} \cdot \nabla_{\mathbf{k}_{c}} f=\left(\frac{\delta f}{\delta t}\right)_{\text {scatt }} .
$$

Here the first term on the left hand side is the derivative with respect to the explicit time dependence of $f$, the next two terms account for the time dependence of $\mathbf{r}_{c}$ and $\mathbf{k}_{c}$, and the term on the right hand side describes scattering of electron states, by defects and other perturbation, $\mathrm{s}$ in and out of the considered phase space volume centered at $\mathbf{r}_{c}$ and $\mathbf{k}_{c}$. The time evolution of $\mathbf{r}_{c}(\mathrm{t})$ and $\mathbf{k}_{c}(\mathrm{t})$ is given by semiclassical equations of motion which in the presence of external electric $\mathbf{E}$ and magnetic $\mathbf{B}$ fields take conventionally the following form

$$
\begin{aligned}
\dot{\mathbf{r}}_{c} & =\left.\frac{1}{\hbar} \frac{\partial \mathcal{E}_{n \mathbf{k}}}{\partial \mathbf{k}}\right|_{\mathbf{k}=\mathbf{k}_{c}}=\mathbf{v}_{n}\left(\mathbf{k}_{c}\right), \\
\hbar \dot{\mathbf{k}}_{c} & =-e \mathbf{E}-e \dot{\mathbf{r}}_{c} \times \mathbf{B} .
\end{aligned}
$$

Here, the group velocity $\mathbf{v}_{n}\left(\mathbf{k}_{c}\right)$ involves the unperturbed band structure. In the presence of a constant electric $\mathbf{E}=\left(E_{x}, 0,0\right)$ and magnetic $\mathbf{B}=\left(0,0, B_{z}\right)$ field the solution of the above equations yields the Hall resistivity $\rho_{x y} \propto B_{z}$. However, for a ferromagnet with magnetization $\mathbf{M}=\left(0,0, M_{z}\right)$ the above theory can not account for the experimentally observed (additional) anomalous contribution $\rho_{x y} \propto M_{z}$. For a necessary correction of the theory, one needs to construct a wave packet

$$
\mathrm{W}_{\mathbf{k}_{c}, \mathbf{r}_{c}}(\mathbf{r}, t)=\sum_{\mathbf{k}} a_{\mathbf{k}}\left(\mathbf{k}_{c}, t\right) \Psi_{n \mathbf{k}}(\mathbf{r})
$$

carefully taking into account the phase of the weighting function $a_{\mathbf{k}}\left(\mathbf{k}_{c}, t\right)$. Requiring the wave packet to be centered at $\mathbf{r}_{c}=\left\langle W_{\mathbf{k}_{c}, \mathbf{r}_{c}}|\mathbf{r}| W_{\mathbf{k}_{c}, \mathbf{r}_{c}}\right\rangle$ and considering zero order in the magnetic field only, it follows that $a_{\mathbf{k}}\left(\mathbf{k}_{c}, t\right)=\left|a_{\mathbf{k}}\left(\mathbf{k}_{c}, t\right)\right| e^{i\left(\mathbf{k}-\mathbf{k}_{c}\right) \cdot \mathbf{A}_{n}\left(\mathbf{k}_{c}\right)}[2]$, where

$$
\mathbf{A}_{n}\left(\mathbf{k}_{c}\right)=i \int_{\text {u.c. }} \mathrm{d}^{3} \mathrm{r} u_{n}^{*}\left(\mathbf{r}, \mathbf{k}_{c}\right) \nabla_{\mathbf{k}} u_{n}\left(\mathbf{r}, \mathbf{k}_{c}\right)
$$

is the Berry connection as will be discussed in detail in the next Section. Note that the integral is restricted to only one unit cell (u.c.).

Now, using the wave packet of Eq. (4), one can construct the Lagrangian

$$
L(t)=\left\langle W\left|i \hbar \partial_{t}-\hat{H}\right| W\right\rangle
$$


and minimize the action functional $\mathrm{S}\left[\mathbf{r}_{c}(t), \mathbf{k}_{c}(t)\right]=\int^{t} d t^{\prime} L\left(t^{\prime}\right)$ with respect to arbitrary variations in $\mathbf{r}_{c}(t)$ and $\mathbf{k}_{c}(t)$. This manoeuvre replaces the quantum mechanical expectation values with respect to $\mathrm{W}_{\mathbf{k}_{c}, \mathbf{r}_{c}}(\mathbf{r}, t)$ by the classical equations of motion for $\mathbf{r}_{c}(t)$ and $\mathbf{k}_{c}(t)$. From the EulerLagrange equations

$$
\partial_{t} \frac{\partial L}{\partial \dot{\mathbf{k}}_{c}}-\frac{\partial L}{\partial \mathbf{k}_{c}}=0 \text { and } \partial_{t} \frac{\partial L}{\partial \dot{\mathbf{r}}_{c}}-\frac{\partial L}{\partial \mathbf{r}_{c}}=0
$$

one gets

$$
\begin{aligned}
\dot{\mathbf{r}_{c}} & =\left.\frac{1}{\hbar} \frac{\partial \mathcal{E}_{n \mathbf{k}}}{\partial \mathbf{k}}\right|_{\mathbf{k}=\mathbf{k}_{c}}-\dot{\mathbf{k}}_{c} \times \boldsymbol{\Omega}_{n}\left(\mathbf{k}_{c}\right) \\
\hbar \dot{\mathbf{k}}_{c} & =-e \mathbf{E}-e \dot{\mathbf{r}}_{c} \times \mathbf{B}
\end{aligned}
$$

Here $\boldsymbol{\Omega}_{n}\left(\mathbf{k}_{c}\right)$ is the so-called Berry curvature, defined as

$$
\boldsymbol{\Omega}_{n}\left(\mathbf{k}_{c}\right)=\nabla_{\mathbf{k}} \times\left.\mathbf{A}_{n}(\mathbf{k})\right|_{\mathbf{k}=\mathbf{k}_{c}},
$$

the main subject of this Review. For simplicity several further terms in Eqs. (8) and (9) were neglected. Additional curvatures according to the time and real space parameters as well as contributions linear in the magnetic field are generally present $[1,2]$. For the purpose of that review we kept the simplest format to introduce the occurrence of the Berry curvature in the equations of motion.

In comparison to Eq. (2), the new equation of motion for $\mathbf{r}_{c}(t)$ given by Eq. (8) has an additional term proportional to the electric field called the anomalous velocity

$$
\mathbf{v}_{n}^{a}\left(\mathbf{k}_{c}\right)=\frac{e}{\hbar} \mathbf{E} \times \boldsymbol{\Omega}_{n}\left(\mathbf{k}_{c}\right) .
$$

The presence of this velocity provides the anomalous contribution to the Hall current $j_{y}^{H}=$ $j_{y}^{H, \uparrow}+j_{y}^{H, \downarrow}$, where the spin-resolved currents may be written as

$$
\begin{aligned}
j_{y}^{H, \uparrow} & =-e \int_{B Z} \frac{\mathrm{d}^{3} \mathrm{k}_{c}}{(2 \pi)^{3}} \dot{r}_{c \uparrow}^{y}\left(\mathbf{k}_{c}\right) f_{\uparrow}\left(\mathbf{k}_{c}\right)=\sigma_{y x}^{\uparrow}\left(M_{z}\right) E_{x} . \\
j_{y}^{H, \downarrow} & =-e \int_{B Z} \frac{\mathrm{d}^{3} \mathrm{k}_{c}}{(2 \pi)^{3}} \dot{r}_{c \downarrow}^{y}\left(\mathbf{k}_{c}\right) f_{\downarrow}\left(\mathbf{k}_{c}\right)=\sigma_{y x}^{\downarrow}\left(M_{z}\right) E_{x} .
\end{aligned}
$$

In that notation $f_{\uparrow}\left(\mathbf{k}_{c}\right)$ and $f_{\downarrow}\left(\mathbf{k}_{c}\right)$ are the spin-resolved distribution functions which are solutions of Eq. (1). Here $\mathbf{r}_{c}$ and $t$ are dropped due to homogenous and steady state conditions. In the limit of no scattering they reduce to the equilibrium distribution functions and only the intrinsic mechanism, governed by the anomalous velocity of Eq. (11), contributes to the Hall component. In general, $j_{y}^{H, \uparrow}$ and $j_{y}^{H, \downarrow}$ have opposite sign because of $\dot{\mathbf{r}}_{c}\left(\mathbf{k}_{c}\right)$. However, the resulting charge current is non-vanishing (see Fig. 1 (a)) due to the fact, that the number of spin-up and spin-down electrons differs in ferromagnets with a finite magnetization $\mathbf{M} \neq 0$. In contrast to the anomalous Hall effect (AHE) in ferromagnets, the absence of the magnetization in normal metals leads to the presence of time inversion symmetry and, as a consequence, $j_{y}^{H, \uparrow}=-j_{y}^{H, \downarrow}$. This provides a vanishing charge current, i.e. Hall voltage, but the existence of a pure spin current as depicted in Fig. 1 (b), which is known as the spin Hall effect (SHE). 


\subsection{Berry phase, connection and curvature of Bloch electrons}

Here we introduce shortly the concept of such relatively novel quantities as the Berry phase, connection, and curvature which arise in case of an adiabatic evolution of a system.

\subsubsection{Adiabatic evolution and the Berry phase}

Let us consider an eigenvalue problem of the following form

$$
\widehat{H}\left(\mathbf{r}, \lambda_{1}, \lambda_{2}, \lambda_{3}\right) \Phi_{n}\left(\mathbf{r} ; \lambda_{1}, \lambda_{2}, \lambda_{3}\right)=\mathcal{E}_{n}\left(\lambda_{1}, \lambda_{2}, \lambda_{3}\right) \Phi_{n}\left(\mathbf{r} ; \lambda_{1}, \lambda_{2}, \lambda_{3}\right)
$$

where $\widehat{H}$ is a differential operator, $\Phi_{n}$ and $\mathcal{E}_{n}$ are the $n$th eigenfunction and eigenvalue, respectively, the vector $\mathbf{r}$ is a variable, and $\lambda_{1}, \lambda_{2}, \lambda_{3}$ are parameters restricted for simplicity to the number of three. A mathematical problem of general interest is the way such eigenfunctions change if the set $\boldsymbol{\lambda}=\left(\lambda_{1}, \lambda_{2}, \lambda_{3}\right)$ changes slowly with time. If it is assumed that the process is adiabatic, that is to say there is no transition from one to another state, then for long it was argued that a state $\Phi_{n}(t=0)$ evolves in time as $\Phi_{n}(t)=\Phi_{n}(t=0)$ $\exp \left(-\frac{i}{\hbar} \int_{0}^{t} d t^{\prime} \mathcal{E}_{n}\left(\lambda_{1}\left(t^{\prime}\right), \lambda_{2}\left(t^{\prime}\right), \lambda_{3}\left(t^{\prime}\right)\right)+i \gamma_{n}(t)\right)$, where the phase $\gamma_{n}(t)$ is meaningless as it can be gauged away. In 1984 M. V. Berry [3] has discovered that while this is true for an arbitrary open path in the parameter space, for a path that returns to the starting values $\lambda_{1}(0), \lambda_{2}(0)$, and $\lambda_{3}(0)$ the accumulated phase is gauge invariant and is therefore a meaningful and measurable quantity. In particular, he showed that for a closed path, labeled $C$ in Fig. 2, the phase $\gamma_{n}$, called Berry

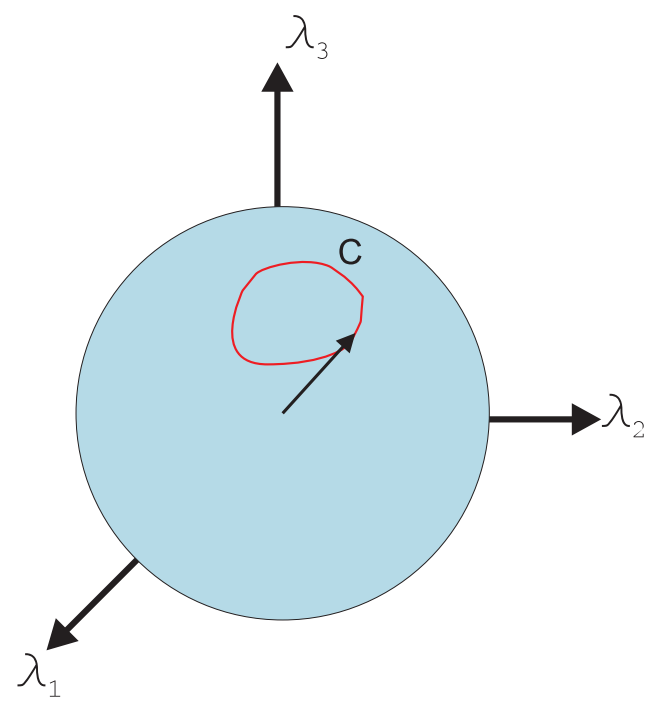

Figure 2: A closed path in the parameter space.

phase today, can be described by a line integral as

$$
\gamma_{n}=\oint_{C} \mathrm{~d} \boldsymbol{\lambda} \cdot \mathbf{A}_{n}(\boldsymbol{\lambda})
$$

where the connection $\mathbf{A}_{n}(\boldsymbol{\lambda})$ is given by

$$
\mathbf{A}_{n}(\boldsymbol{\lambda})=i \int \mathrm{d}^{3} \mathrm{r} \Phi_{n}^{*}(\mathbf{r}, \boldsymbol{\lambda}) \nabla_{\boldsymbol{\lambda}} \Phi_{n}(\mathbf{r}, \boldsymbol{\lambda})
$$


The surprise is that although the connection is not gauge invariant, $\gamma_{n}$ and even more interestingly the curvature

$$
\boldsymbol{\Omega}_{n}(\boldsymbol{\lambda})=\nabla_{\boldsymbol{\lambda}} \times \mathbf{A}_{n}(\boldsymbol{\lambda})
$$

are. As will be shown presently, these ideas are directly relevant for the dynamics of wave packets in the transport theory discussed above.

\subsubsection{Berry connection and curvature for Bloch states}

Somewhat surprisingly the above discussion has a number of interesting things to say about the wave packets in the semiclassical approach for spin and charge transport of electrons in crystals. Its relevance becomes evident if we take the Schrödinger equation for the Bloch state

$$
\left(-\frac{\hbar^{2}}{2 m} \nabla_{\mathbf{r}}^{2}+V(\mathbf{r})\right) \Psi_{n \mathbf{k}}(\mathbf{r})=\mathcal{E}_{n \mathbf{k}} \Psi_{n \mathbf{k}}(\mathbf{r})
$$

and rewrite it for the periodic part of the Bloch function

$$
\left(-\frac{\hbar^{2}}{2 m}\left(\nabla_{\mathbf{r}}+i \mathbf{k}\right)^{2}+V(\mathbf{r})\right) u_{n}(\mathbf{r}, \mathbf{k})=\mathcal{E}_{n \mathbf{k}} u_{n}(\mathbf{r}, \mathbf{k}) .
$$

The point is that the differential equation for $\Psi_{n \mathbf{k}}(\mathbf{r})$, a simultaneous eigenfunction of the translation operator and the Hamiltonian, does not depend on the wave vector, since $\mathbf{k}$ only labels the eigenvalues of the translation operator. However, in Eq. (19) $\mathbf{k}$ appears as a parameter and hence the above discussion of the Berry phase, the corresponding connection, and curvature applies directly to the periodic part of the wave function $u_{n}(\mathbf{r}, \mathbf{k})$. In short, for each band $n$ there is a connection associated with every $\mathbf{k}$ given by

$$
\mathbf{A}_{n}(\mathbf{k})=i \int_{\text {u.c. }} \mathrm{d}^{3} \mathrm{r} u_{n}^{*}(\mathbf{r}, \mathbf{k}) \nabla_{\mathbf{k}} u_{n}(\mathbf{r}, \mathbf{k}) .
$$

Furthermore, the curvature

$$
\boldsymbol{\Omega}_{n}(\mathbf{k})=\nabla_{\mathbf{k}} \times \mathbf{A}_{n}(\mathbf{k})=i \int_{\text {u.c. }} \mathrm{d}^{3} \mathrm{r} \nabla_{\mathbf{k}} u_{n}^{*}(\mathbf{r}, \mathbf{k}) \times \nabla_{\mathbf{k}} u_{n}(\mathbf{r}, \mathbf{k})
$$

is the quantity that appears in the semiclassical equation of motion for the wave packet in Eq. (8). Thus, the curvature and the corresponding anomalous velocity in Eq. (11) associated with the band $n$ are properties of $u_{n}(\mathbf{r}, \mathbf{k})$. To emphasize this point, we note that the Bloch functions $\Psi_{n \mathbf{k}}(\mathbf{r})$ are orthogonal to each other

$$
\left\langle\Psi_{n \mathbf{k}} \mid \Psi_{n^{\prime} \mathbf{k}^{\prime}}\right\rangle=\int_{\text {crystal }} \mathrm{d}^{3} \mathrm{r} \Psi_{n \mathbf{k}}^{*}(\mathbf{r}) \Psi_{n^{\prime} \mathbf{k}^{\prime}}(\mathbf{r})=\delta_{\mathbf{k}, \mathbf{k}^{\prime}} \delta_{n, n^{\prime}},
$$

and hence $\mathbf{k}$ is not a parameter. In contrast, $u_{n}(\mathbf{r}, \mathbf{k})$ is orthogonal to $u_{n^{\prime}}(\mathbf{r}, \mathbf{k})$ but not necessarily to $u_{n}\left(\mathbf{r}, \mathbf{k}^{\prime}\right)$ as this is an eigenfunction of a different Hamiltonian.

The observation that the above connection and curvature are properties of the periodic part of the Bloch function prompts a further comment. Note, that for vanishing periodic potential the Bloch functions would reduce to plane waves. Constructing the wave packet from plane waves means $u_{n}(\mathbf{r}, \mathbf{k})=1$. Evidently, this means that $\boldsymbol{\Omega}_{n}(\mathbf{k})$ and $\mathbf{A}_{n}(\mathbf{k})$ vanish and this fact is 
consistent with the general notion that the connection and the curvature arise from including only one or in any case finite number of bands, in constructing the wave packet. The point is that the neglected bands are the 'outside world' referred to in the original article of M. V. Berry [3]. In contrast, the expansion in terms of plane waves does not neglect any bands since there is just one band. Thus, the physical origin of the connection and the curvature is due to the periodic crystal potential breaking up the full spectrum of the parabolic free particle dispersion relation into bands. They are usually separated by gaps, and we select states only from a limited number of such bands to represent the wave packet.

\subsection{Abelian and non-Abelian curvatures}

So far we have introduced the Berry connection and curvature for a non-degenerate band. In case of degenerate bands the conventional adiabatic theorem fails. In the semiclassical framework a correct treatment incorporates a wave packet constructed from the degenerate bands $[4,5]$. As a consequence, the Berry curvature must be extended to a tensor definition in analogy to non-Abelian gauge theories. This extension is originally due to Wilczek and Zee [6].

Let us consider the eigenspace $\left\{\left|u_{i}(\mathbf{k})\right\rangle: i \in \Sigma\right\}$ of some $N$-fold degenerate eigenvalue. For Bloch states the elements of the non-Abelian Berry connection $\overline{\overline{\mathbf{A}}}(\mathbf{k})$ read

$$
\mathbf{A}_{i j}(\mathbf{k})=i\left\langle u_{i}(\mathbf{k}) \mid \nabla_{\mathbf{k}} u_{j}(\mathbf{k})\right\rangle \quad i, j \in \Sigma,
$$

where $\Sigma=\{1, \ldots, N\}$ contains all indices of the degenerate subspace. The definition of the curvature tensor $\overline{\bar{\Omega}}(\mathbf{k})$ in a non-Abelian gauge theory has to be extended by substituting the curl with the covariant derivative as [5]

$$
\begin{aligned}
\overline{\overline{\mathbf{\Omega}}}(\mathbf{k}) & =\boldsymbol{\nabla}_{\mathbf{k}} \times \overline{\overline{\mathbf{A}}}(\mathbf{k})-i \overline{\overline{\mathbf{A}}}(\mathbf{k}) \times \overline{\overline{\mathbf{A}}}(\mathbf{k}) \\
\boldsymbol{\Omega}_{i j}(\mathbf{k}) & =i\left\langle\frac{\partial u_{i}(\mathbf{k})}{\partial \mathbf{k}}|\times| \frac{\partial u_{j}(\mathbf{k})}{\partial \mathbf{k}}\right\rangle+i \sum_{l \in \Sigma}\left\langle u_{i}(\mathbf{k}) \mid \frac{\partial u_{l}(\mathbf{k})}{\partial \mathbf{k}}\right\rangle \times\left\langle u_{l}(\mathbf{k}) \mid \frac{\partial u_{j}(\mathbf{k})}{\partial \mathbf{k}}\right\rangle .
\end{aligned}
$$

The subspace of degenerate eigenstates is subject to a $\mathrm{U}(N)$ gauge freedom and observables should be invariant with respect to a gauge transformation of the basis set

$$
\left\{\sum_{i} U_{i j}(\mathbf{k})\left|u_{i}(\mathbf{k})\right\rangle: i \in \Sigma\right\} \quad \text { with } \overline{\bar{U}}(\mathbf{k}) \in \mathrm{U}(N) \text {. }
$$

Consequently, the Berry connection and curvature are transformed according to

$$
\begin{aligned}
\overline{\overline{\mathbf{A}}}^{\prime}(\mathbf{k}) & =\overline{\bar{U}}^{\dagger}(\mathbf{k}) \overline{\overline{\mathbf{A}}}(\mathbf{k}) \overline{\bar{U}}(\mathbf{k})+i \overline{\bar{U}}^{\dagger}(\mathbf{k}) \boldsymbol{\nabla}_{\mathbf{k}} \overline{\bar{U}}(\mathbf{k}) \\
\overline{\overline{\mathbf{\Omega}}}^{\prime}(\mathbf{k}) & =\overline{\bar{U}}^{\dagger}(\mathbf{k}) \overline{\overline{\mathbf{\Omega}}}(\mathbf{k}) \overline{\bar{U}}(\mathbf{k}) .
\end{aligned}
$$

The curvature is now a covariant tensor and thus not directly observable. However, there are gauge invariant quantities that may be derived from it. From gauge theories it is known that the connection and the curvature must lie in the tangent space of the gauge group $\mathrm{U}(N)$, i.e., the Lie algebra $\mathfrak{u}(N)$. The above definition of the Lie algebra differs by an unimportant factor of $i$ from the mathematical convention.

The most prominent example of a non-Abelian Berry curvature appears in materials with timereversal (T) and inversion (I) symmetry. For each $\mathbf{k}$ point there exist two orthogonal states 
$\left|\Psi_{n \uparrow \mathbf{k}}\right\rangle$ and $\left|\Psi_{n \downarrow \mathbf{k}}\right\rangle=\mathrm{TI}\left|\Psi_{n \uparrow \mathbf{k}}\right\rangle$ with the same energy, the Kramers doublet. In general, a choice for $\left|\Psi_{n \uparrow \mathbf{k}}\right\rangle$ determines the second basis state $\left|\Psi_{n \downarrow \mathbf{k}}\right\rangle$ only up to a phase. However, the symmetry transformation fixes the phase relationship between both basis states. This reduces the gauge freedom to SU(2) and, consequently, the Berry connection will be an $\mathfrak{s u}(2)$ matrix, which means its trace vanishes. This is verified easily

$$
\begin{aligned}
\left\langle u_{n \downarrow}(\mathbf{k}) \mid \nabla_{\mathbf{k}} u_{n \downarrow}(\mathbf{k})\right\rangle & =\left\langle\operatorname{TI} u_{n \uparrow}(\mathbf{k}) \mid \nabla_{\mathbf{k}} \mathrm{TI} u_{n \uparrow}(\mathbf{k})\right\rangle=\left\langle\operatorname{TI} u_{n \uparrow}(\mathbf{k}) \mid \operatorname{TI} \nabla_{\mathbf{k}} u_{n \uparrow}(\mathbf{k})\right\rangle \\
& =-\left\langle u_{n \uparrow}(\mathbf{k}) \mid \nabla_{\mathbf{k}} u_{n \uparrow}(\mathbf{k})\right\rangle .
\end{aligned}
$$

For the Kramers doublet the Berry curvature must always be an $\mathfrak{s u}(2)$ matrix even in the case of a $\mathfrak{u}(2)$ connection because a gauge transformation induces a unitary transformation of the curvature which does not alter the trace. Indeed, $\operatorname{TI} \boldsymbol{\Omega}_{n \uparrow \uparrow}(\mathbf{k})=-\boldsymbol{\Omega}_{n \uparrow \uparrow}(\mathbf{k})=\boldsymbol{\Omega}_{n \downarrow \downarrow}(\mathbf{k})$, which proves that the trace is equal zero. Therefore, in contrast to the spinless case, discussed in the next section, the Berry curvature may be nontrivial even in nonmagnetic materials with inversion symmetry.

Despite the Berry curvature being gauge covariant we may derive several observables from it. As we have seen the trace of the Berry curvature is gauge invariant. In the multiband formulation of the semiclassical theory, the expectation value of the curvature matrix with respect to the spinor amplitudes enters the equations of motion [4, 5]. In the context of the spin Hall effect one is interested in $\operatorname{Tr}\left(\overline{\bar{S}}^{j} \overline{\bar{\Omega}}^{i}\right)$, where $\overline{\bar{S}}^{j}$ is the $j$-th component of the vector valued $\mathfrak{s u}(2)$ spin matrix (cf. Sec. 2.4.2) .

\subsection{Symmetry, topology, codimension and the Dirac monopole}

It is expedient to exploit symmetries of the Hamiltonian for the evaluation of the Berry curvature. From Eqs. (20) and (21) we may easily determine the behavior of $\boldsymbol{\Omega}_{n}(\mathbf{k})$ under symmetry operations. In crystals with a center of inversion, the corresponding symmetry operation leaves $\mathcal{E}_{n \mathbf{k}}$ invariant while $\mathbf{r}, \dot{\mathbf{r}}, \mathbf{k}$, and $\dot{\mathbf{k}}$ change sign, and hence $\boldsymbol{\Omega}_{n}(\mathbf{k})=\boldsymbol{\Omega}_{n}(-\mathbf{k})$. On the other hand, when time-reversal symmetry is present $\mathcal{E}_{n \mathbf{k}}, \mathbf{r}$, and $\dot{\mathbf{k}}$ remain unchanged while $\dot{\mathbf{r}}$ and $\mathbf{k}$ are inverted, which leads to an antisymmetric Berry curvature $\boldsymbol{\Omega}_{n}(\mathbf{k})=-\boldsymbol{\Omega}_{n}(-\mathbf{k})$. Thus, if time-reversal and inversion symmetry are present simultaneously the Berry curvature vanishes identically [7]. This is true for spinless particles only. Taking into account spin we have to acknowledge the presence of a twofold degeneracy of all bands throughout the Brillouin zone $[8,9]$, the Kramers doublet, discussed in the previous section.

As was mentioned already in section 1.2.2, the Berry curvature of a band arises due to the attempt of a single band description, e.g., in the semiclassical theory it keeps the information about the influence of other adjacent bands. If a band is well separated from all other bands by an energy scale large compared to one set by the time scale of the adiabatic evolution, the influence of the other bands is negligible. In contrast, degeneracies of energy bands deserve special attention, since the conventional adiabatic theorem fails in this case. Special attention has been paid to point-like degeneracies, where the intersection of two energy bands is shaped like a double cone or a diabolo. These degeneracies are called diabolical points [10]. A typical one, studied within a tight-binding model in Ref. [11], is shown in Fig. 3.

In general a Hermetian Hamiltonian $\hat{H}(\mathbf{k})$ with three parameters $k_{x}, k_{y}, k_{z}$ has degeneracies (band 

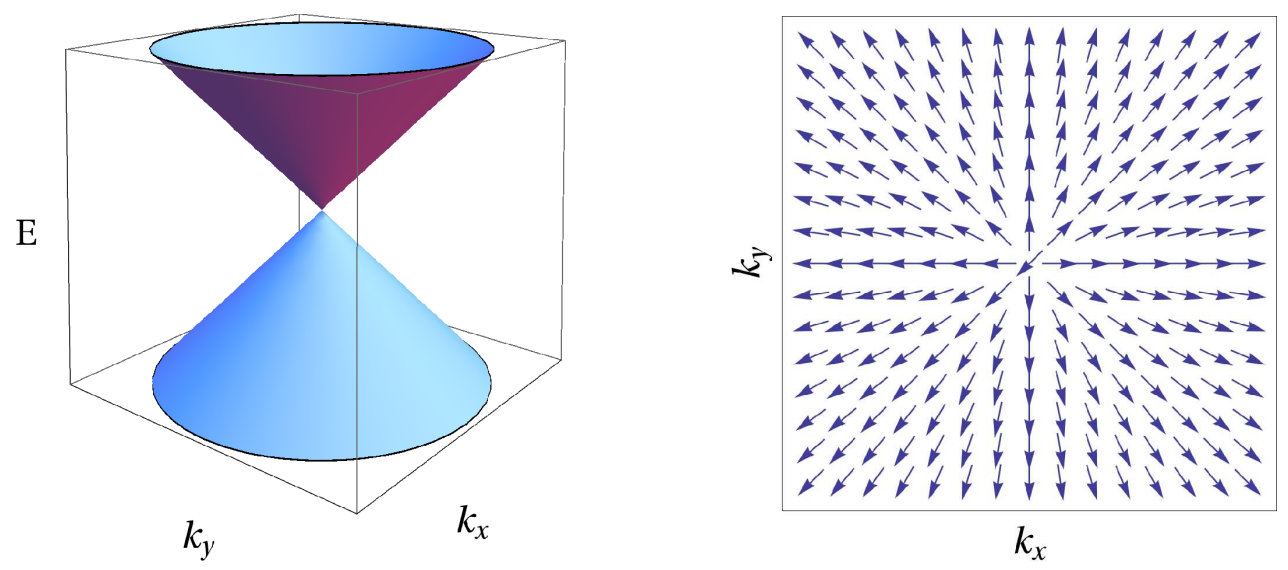

Figure 3: Left: Conical intersection of energy surfaces. Right: Direction of the Monopole field $\Omega_{+}(\mathbf{k})$.

crossings) at points $\mathbf{k}^{*}$. Taking $\mathbf{k}^{*}$ as the origin and assuming that $\hat{H}(\mathbf{k})$ depends linearly on the $\mathbf{k}$ measured from $\mathbf{k}^{*}$, a generic example for two bands crossing can be constructed as follows:

At $\mathbf{k}=\mathbf{k}^{*}$ there are two orthogonal states $|a\rangle$ and $|b\rangle$ whose energies are the same $\mathcal{E}_{a}=\mathcal{E}_{b}=0$ which we take to be the energy zero. In the vicinity of the point $\mathbf{k}^{*}$ we can express the eigenstates in terms of the two states

$$
\left|u_{n}(\mathbf{k})\right\rangle=c_{a n}(\mathbf{k})|a\rangle+c_{b n}(\mathbf{k})|b\rangle
$$

where $n=\{+,-\}$ and the coefficients $c_{a n}(\mathbf{k})$ and $c_{b n}(\mathbf{k})$ are determined by the eigenvalue problem

$$
\hat{H}(\mathbf{k})\left|u_{n}(\mathbf{k})\right\rangle=\mathcal{E}_{n \mathbf{k}}\left|u_{n}(\mathbf{k})\right\rangle
$$

Using the expansion of Eq. (29) little is lost in generality by taking $\hat{H}(\mathbf{k})$ in the representation of the two states $|a\rangle$ and $|b\rangle$ to be of the form [3]

$$
H(\mathbf{k})=\left(\begin{array}{cc}
k_{z} & k_{x}-i k_{y} \\
k_{x}+i k_{y} & -k_{z}
\end{array}\right)
$$

and hence the eigenvalue equation can written as

$$
\left(\begin{array}{cc}
k_{z} & k_{x}-i k_{y} \\
k_{x}+i k_{y} & -k_{z}
\end{array}\right)\left(\begin{array}{c}
c_{a n}(\mathbf{k}) \\
c_{b n}(\mathbf{k})
\end{array}\right)=\mathcal{E}_{n \mathbf{k}}\left(\begin{array}{c}
c_{a n}(\mathbf{k}) \\
c_{b n}(\mathbf{k})
\end{array}\right) .
$$

The solution of this eigenvalue problem is

$$
\mathcal{E}_{ \pm \mathbf{k}}= \pm \sqrt{k_{z}^{2}+k_{x}^{2}+k_{y}^{2}}= \pm|\mathbf{k}|= \pm k
$$

and the two eigenvectors are

$$
\begin{aligned}
u_{+}(\mathbf{k}) \equiv\left(\begin{array}{c}
c_{a+}(\mathbf{k}) \\
c_{b+}(\mathbf{k})
\end{array}\right) & =\left(\begin{array}{c}
\sqrt{\frac{k+k_{z}}{2 k}} e^{-i \frac{\delta_{+}}{2}} \\
\sqrt{\frac{k-k_{z}}{2 k}} e^{+i \frac{\delta_{+}}{2}}
\end{array}\right) \\
\delta_{+} & =\tan ^{-1}\left(\frac{k_{y}}{k_{x}}\right)
\end{aligned}
$$




$$
\begin{aligned}
u_{-}(\mathbf{k}) \equiv\left(\begin{array}{c}
c_{a-}(\mathbf{k}) \\
c_{b-}(\mathbf{k})
\end{array}\right) & =\left(\begin{array}{c}
\sqrt{\frac{k-k_{z}}{2 k}} e^{-i \frac{\delta_{-}}{2}} \\
-\sqrt{\frac{k+k_{z}}{2 k}} e^{+i \frac{\delta_{-}}{2}}
\end{array}\right) \\
\delta_{-} & =\tan ^{-1}\left(\frac{k_{y}}{k_{x}}\right)
\end{aligned}
$$

where we used the shortcut $u_{ \pm}(\mathbf{k})$ for the two component eigenvectors representing the expansion coefficients of $\left|u_{n}(\mathbf{k})\right\rangle$. Clearly, $\delta_{+}=\delta_{-} \equiv \delta$ holds.

Calculating the connection

$$
\mathbf{A}_{+}(\mathbf{k})=i u_{+}^{\dagger}(\mathbf{k}) \nabla_{\mathbf{k}} u_{+}(\mathbf{k})
$$

and the curvature as $[3]$

$$
\boldsymbol{\Omega}_{+}(\mathbf{k})=\operatorname{Im} \frac{u_{+}^{\dagger}(\mathbf{k}) \boldsymbol{\nabla}_{\mathbf{k}} \hat{H}(\mathbf{k}) u_{-}(\mathbf{k}) \times u_{-}^{\dagger}(\mathbf{k}) \boldsymbol{\nabla}_{\mathbf{k}} \hat{H}(\mathbf{k}) u_{+}(\mathbf{k})}{\left(\mathcal{E}_{+\mathbf{k}}-\mathcal{E}_{-\mathbf{k}}\right)^{2}}
$$

one finds

$$
\boldsymbol{\Omega}_{ \pm}(\mathbf{k})= \pm \frac{1}{2} \frac{\mathbf{k}}{k^{3}}
$$

This is precisely the form of the magnetic field due to a Dirac monopole with the magnetic charges $\pm \frac{1}{2}$ [3] (see Fig. 3, right). Diabolical points always have this charge, while nonlinear intersections may have higher charges [12]. In general, the monopole charge is a topological invariant and quantized to integer multiples of $1 / 2$. This is a consequence of the quantization of the Dirac monopole [13].

In more general language, the Berry curvature flux through a closed two-dimensional manifold $\partial M$ is the first Chern number $C_{n}$ of that manifold and thus an integer. Since the (Abelian) curvature is defined as the curl of the connection it must be divergence free except for the monopoles. We can then exploit Gauss' theorem

$$
C_{n}=\frac{1}{2 \pi} \int_{\partial M} \mathrm{~d} \sigma \boldsymbol{\Omega}_{n}(\mathbf{k}) \mathbf{n}(\mathbf{k})=\frac{1}{2 \pi} \int_{M} \mathrm{~d}^{3} \mathrm{k} \boldsymbol{\nabla}_{\mathbf{k}} \cdot \boldsymbol{\Omega}_{n}=m, \quad m \in \mathbb{N} .
$$

Here $\mathbf{n}$ is the normal vector of the surface element. If we assume that each monopole contributes a Chern number of \pm 1 the above equation implies for the Berry curvature $\nabla_{\mathbf{k}} \cdot \boldsymbol{\Omega}(\mathbf{k})=$ $4 \pi \sum_{j} g_{j} \delta\left(\mathbf{k}-\mathbf{k}_{j}^{*}\right)$, where $g_{j}= \pm 1 / 2$. The solution of this differential equation yields the source term of the Berry curvature

$$
\boldsymbol{\Omega}(\mathbf{k})=\sum_{j} g_{j} \frac{\mathbf{k}-\mathbf{k}_{j}^{*}}{\left|\mathbf{k}-\mathbf{k}_{j}^{*}\right|^{3}}+\nabla_{\mathbf{k}} \times \mathbf{A}(\mathbf{k}),
$$

which proves that there are monopoles with a charge quantized to $1 / 2$ times an integer.

The mathematical interpretation of the Berry curvature in terms of the theory of fiber bundles and Chern classes and the connection to the theory of the quantum Hall effect, where the Chern number appears as the Thouless-Kohmoto-Nightingale-den Nijs (TKNN) integer [14] has been first noticed by Simon [15].

However, monopoles are not only interesting in a mathematical sense but also for actual physical quantities such as the anomalous Hall conductivity. On the one hand, singularities of the Berry curvature near the Fermi surface greatly influence the numerical stability of the result. On the other hand, Haldane [16] has pointed out that the Chern number and thus the anomalous Hall conductivity can be changed by creation and annihilation of Berry curvature monopoles. 
To identify monopoles in a parameter space one is interested in degeneracies that do not occur on account of any symmetry and have thus been called accidental. For a single Hamiltonian without symmetries the occurrence of degeneracies in the discrete spectrum is infinitely unlikely. For Hamiltonians that depend on a set of parameters one can ask how many parameters are needed in order to encounter a twofold degenerate eigenvalue. This number is called codimension. According to a theorem by von Neumann and Wigner [17], for a generic Hamiltonian the answer is three. In other words, degeneracies are points in a three-dimensional parameter space like the Brillouin zone. For this reason accidental degeneracies are diabolical points.

If the Hamiltonian is real, which is equivalent to being invariant with respect to time reversal, the number of parameters we have to vary reduces to two [18]. However, we have seen that in the case of nonmagnetic crystals with inversion symmetry each band is twofold degenerate. This degeneracy arises due to a symmetry and is not accidental. The theorem of von Neumann and Wigner does not apply. As a consequence, accidental degeneracies of two different bands would have to be fourfold. In this case the codimension is five and the occurrence of an accidental degeneracy is infinitely unlikely in the Brillouin zone.

This is the reason why we only observe avoided crossings for nonmagnetic crystals with inversion symmetry. All accidental crossings are lifted by spin-orbit coupling. In order to encounter accidental two-fold degeneracies of codimension two, we would, thus, need to consider a nonmagnetic band structure without inversion symmetry of the lattice.

\subsection{The spin polarization gauge}

As has been discussed in the previous sections, in spinless crystals with time-reversal and inversion symmetry the Berry curvature vanishes at every $\mathbf{k}$ point [7]. If spin degrees of freedom are taken into account the band structure is twofold degenerate throughout the Brillouin zone due to Kramers degeneracy plus inversion symmetry and consequently the Berry curvature is represented by an element of the Lie algebra $\mathfrak{s u}(2)$. In contrast to the Abelian case, this matrix is gauge covariant and hence not observable. However, at least two gauge invariant quantities can be derived from the Berry curvature matrix. First of all the trace is gauge invariant but equal zero at all $\mathbf{k}$ points in the considered situation of nonmagnetic inversion symmetric systems. In addition, the determinant of the covariant matrix is gauge invariant which might be used to visualize the non-Abelian Berry curvature. Since this quantity is gauge invariant it should be observable. In general, a correct non-Abelian theory for physical observables must be derived to make use of the full Berry curvature matrix. Nevertheless a simplified single band picture, where just the diagonal elements of the Berry curvature are used, might be helpful if an approximative theory is applied. Then a certain gauge has to be chosen. For example, in case of the spin Hall conductivity it was shown [19] that the diagonal element of the Berry curvature in a certain gauge is sufficient to well approximate full quantum mechanical approaches. With respect to first principles calculations the necessary choice of gauge was discussed in the literature $[20,21,22]$. The idea of this section is to discuss two possible gauges and to compare their implications on matrix quantities as the spin polarization and the Berry curvature.

One natural choice of a gauge, let us call it gauge I, is to ensure that the spin polarization of 
one Kramers state $\mid \psi_{\uparrow, \mathbf{k}}>$ is parallel to the $z$ direction and positive [22], that is

$$
\begin{aligned}
& \left\langle\Psi_{\uparrow \mathbf{k}}\left|\sigma_{x}\right| \Psi_{\uparrow \mathbf{k}}\right\rangle=0, \\
& \left\langle\Psi_{\uparrow \mathbf{k}}\left|\sigma_{y}\right| \Psi_{\uparrow \mathbf{k}}\right\rangle=0, \\
& \left\langle\Psi_{\uparrow \mathbf{k}}\left|\sigma_{z}\right| \Psi_{\uparrow \mathbf{k}}\right\rangle>0 .
\end{aligned}
$$

This means for the second Kramers $\mid \psi_{\downarrow}, \mathbf{k}>$ state

$$
\left\langle\Psi_{\downarrow \mathbf{k}}\left|\sigma_{z}\right| \Psi_{\downarrow \mathbf{k}}\right\rangle=-\left\langle\Psi_{\uparrow \mathbf{k}}\left|\sigma_{z}\right| \psi_{\uparrow \mathbf{k}}\right\rangle<0 .
$$

More generally, we will discuss the spin polarization as an $\mathfrak{s u}(2)$ matrix $\left(\overline{\overline{\mathbf{S}}}_{n}(\mathbf{k})\right)_{i j}=\left\langle\Psi_{i \mathbf{k}}|\boldsymbol{\sigma}| \Psi_{j \mathbf{k}}\right\rangle$ with $i, j=\uparrow, \downarrow$, as it appears in the context of the spin Hall effect. Conditions (43) then imply that the diagonal elements are aligned along the $z$ direction in this gauge. The positiveness of the first diagonal element is essential, since in this way we can distinguish the two degenerate states. However, this fails when the spin polarization goes to zero within this gauge.

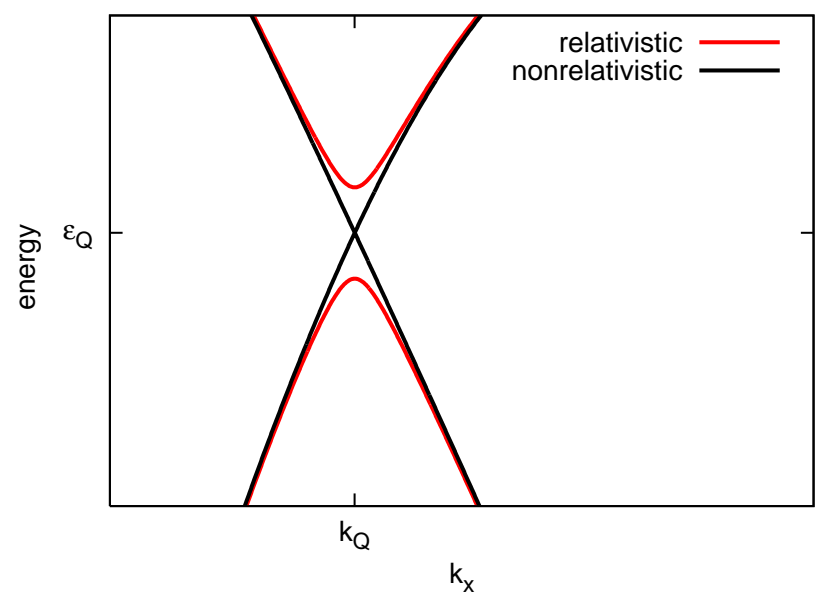

Figure 4: Band structure with (red) and without (black) spin-orbit coupling around point Q obtained within tight-binding calculations [11]. The fourfold accidental degeneracy of the nonmagnetic band structure is lifted.

As a result of the theorem by Wigner and von Neumann [17] discussed in section 1.4, we do not expect accidental degeneracies in nonmagnetic materials. They are lifted by spin-orbit coupling. When two bands get close at some point in the Brillouin zone they "repel" each other depending on the strength of spin-orbit coupling. The lifting of accidental degeneracies by spin-orbit coupling, at a certain point $Q$ in momentum space, is illustrated in Fig. 4 with and without spin-orbit interaction is displayed in red and black, respectively. Similar to real crossings in the magnetic case, there are peaks of the Berry curvature at these points, since the two degenerate bands under consideration are significantly influenced by the neighboring bands. However, in contrast to the magnetic case, the curvature remains finite.

When introducing gauge I, we remarked that it is necessary to enforce a positive sign of $\left\langle\Psi_{\uparrow \mathbf{k}}\left|\sigma_{z}\right| \Psi_{\uparrow \mathbf{k}}\right\rangle$ in addition to the alignment of the spin polarization along the $z$ axis. By doing so one can distinguish the two degenerate Kramers states $\left|\Psi_{\uparrow \mathbf{k}}\right\rangle$ and $\left|\Psi_{\downarrow \mathbf{k}}\right\rangle$ according to their spin polarization. This criterion becomes useless once there is a point where the spin polarization vanishes in the chosen gauge. This is exactly the case when the avoided crossing originates 

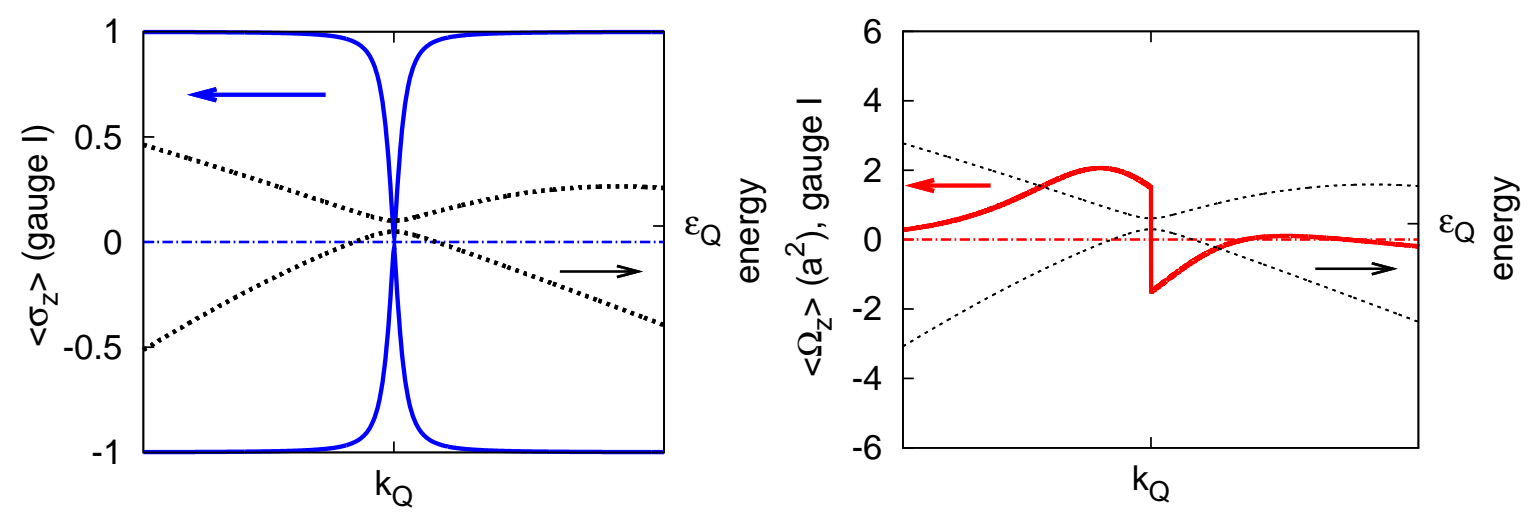

Figure 5: The band structure of two bands (black dotted lines) near an avoided crossing at point $\mathrm{Q}$ in momentum space (see Fig. 4). Left: Both diagonal elements of the $\mathfrak{s u}(2)$ spin polarization (blue full lines) of the Kramers doublet of the lower band in gauge I. Right: The first diagonal element of the $\mathfrak{s u}(2)$ Berry curvature (red full lines) of the same band jumps in gauge I.

from an accidental degeneracy lifted by spin-orbit coupling.

This situation is illustrated in Fig. 5, where the spin polarization for both Kramers states of the lower doublet is shown. The spin polarization becomes zero at the avoided crossing. In fact, the picture suggests that the spin polarizations of both states change sign, which means that by enforcing gauge I we have changed from the first to the second Kramers state while crossing point $Q$. Indeed, this is verified by the graph of the first diagonal element of the Berry curvature in this gauge presented in Fig. 5. Exactly at the point where the spin polarization vanishes, the Berry curvature changes sign, jumping from one degenerate state of the band to the other. This jump is unsatisfactory, since it does not allow us to follow the Berry curvature consistently throughout the BZ without adjustment by hand. A way out is provided by a different gauge. This new gauge (let us call it gauge II) describes the nonmagnetic crystal as the limit of vanishing exchange splitting in a magnetic crystal. The task is then to find the unitary transformation that diagonalizes the perturbation operator $H_{\mathrm{xc}}$ in the degenerate subspace of the Kramers doublet. This amounts to the condition

$$
\left\langle\Psi_{\uparrow \mathbf{k}}\left|\mathcal{H}_{\mathrm{xc}}\right| \Psi_{\downarrow \mathbf{k}}\right\rangle \propto\left\langle\Psi_{\uparrow \mathbf{k}}\left|\sigma_{z}\right| \Psi_{\downarrow \mathbf{k}}\right\rangle=0 .
$$

The above equation accounts for the two free parameters we have to fix. Furthermore, we choose the sign as

$$
\left\langle\Psi_{\uparrow \mathbf{k}}\left|\sigma_{z}\right| \Psi_{\uparrow \mathbf{k}}\right\rangle>0 .
$$

In gauge II the diagonal elements of the nonmagnetic spin polarization represent the analytical continuation of the magnetic spin polarizations with vanishing exchange splitting.

In Fig. 6 the same avoided crossing as in Fig. 5 is shown while gauge II has been imposed. The spin polarization now remains finite and the Berry curvature is continuous, thus we have resolved the ambiguity. This result is clearly an advantage of gauge II in comparison to gauge I. Furthermore, it can be shown that gauge II maximizes the diagonal element of the $\sigma_{z}$ operator [23] with respect to all possible gauges. This implies that for a proper discussion of spin hot spots, the vanishing of the spin expectation value, gauge II has to be chosen. A comparative 

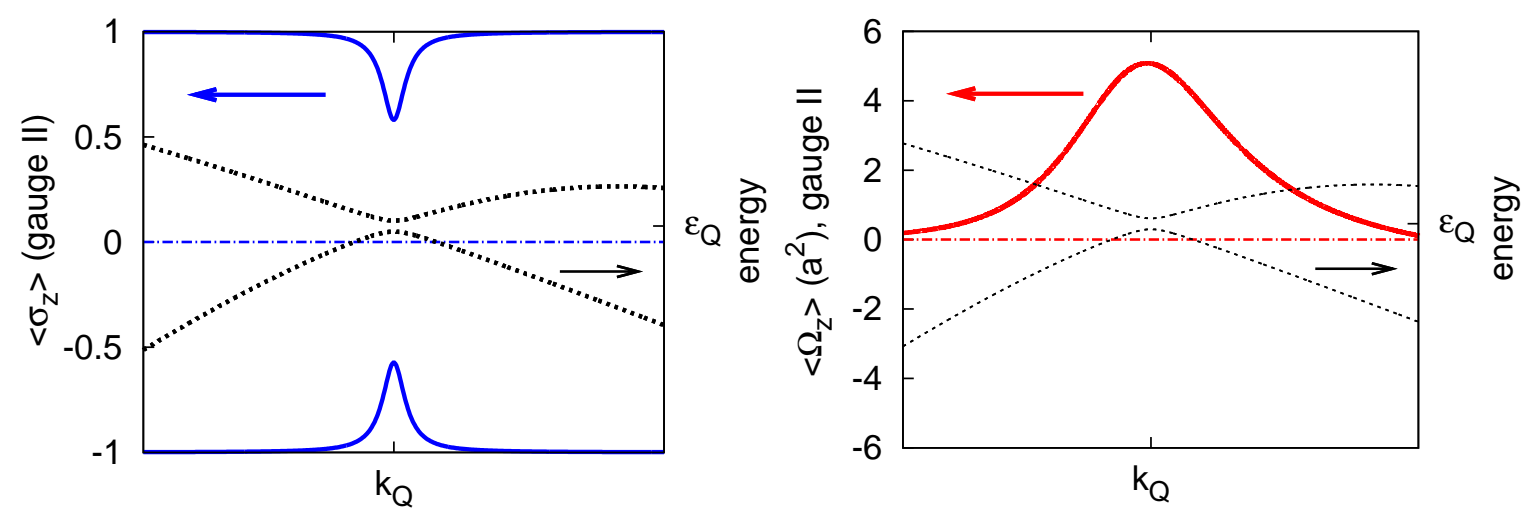

Figure 6: The band structure of two bands (black dotted lines) near an avoided crossing at point $\mathrm{Q}$ in momentum space (see Fig. 4). Left: Both diagonal elements of the nonmagnetic spin polarization (blue) of the lower doublet in gauge II. Right: The first diagonal element of the $\mathfrak{s u}(2)$ Berry curvature (red) remains continuous in gauge II.

study of the implications of the gauge choice in actual first principle calculation will be presented elsewhere [23].

\section{First principle calculations of the Berry curvature for Bloch Electrons}

\section{$2.1 \quad$ KKR method}

\subsubsection{Berry connection}

In this Section we describe the most recent of four methods developed for calculating the Berry curvature within first-principles computational schemes. This approach [19] is based on the screened version of the venerable Korringa-Kohn-Rostoker (KKR) method [24, 25] and is motivated by the fact that in the multiple scattering theory the wave vector $\mathbf{k}$ enters the problem only through the structure constants $G_{Q Q^{\prime}}^{s}(\mathcal{E} ; \mathbf{k})$. Those depend on the geometrical arrangement of the scatterers but not on the scattering potentials at the lattice sites. Hence the sort of $\mathbf{k}$ derivatives that occur in the definition of the connection in Eq. (20) should involve only the derivative $\nabla_{\mathbf{k}} G_{Q Q^{\prime}}^{s}(\mathcal{E} ; \mathbf{k})$ easy to calculate within the screened KKR method. In what follows we demonstrate that such expectation can indeed be realized and an efficient algorithm is readily constructed which takes full advantage of these features.

As it is clear from Eqs. (20) and (21), the connection $\mathbf{A}_{n}(\mathbf{k})$ and the curvature $\boldsymbol{\Omega}_{n}(\mathbf{k})$ are the properties of the periodic component $u_{n}(\mathbf{r}, \mathbf{k})$ of the Bloch state $\Psi_{n \mathbf{k}}(\mathbf{r})$. However, as in most band theory methods, in the KKR one calculates the Bloch wave. Thus, to facilitate the calculation of $\mathbf{A}_{n}(\mathbf{k})$ and $\boldsymbol{\Omega}_{n}(\mathbf{k})$ from $\Psi_{n \mathbf{k}}(\mathbf{r})$ one must recast the problem. A useful way to proceed 
with it is to rewrite

$$
\begin{aligned}
\mathbf{A}_{n}(\mathbf{k}) & =i \int_{\text {u.c. }} \mathrm{d}^{3} \mathrm{r} u_{n}^{\dagger}(\mathbf{r}, \mathbf{k}) \nabla_{\mathbf{k}} u_{n}(\mathbf{r}, \mathbf{k}) \\
& =i \int_{\text {u.c. }} \mathrm{d}^{3} \mathrm{r} \Psi_{n \mathbf{k}}^{*}(\mathbf{r}) \nabla_{\mathbf{k}} \Psi_{n \mathbf{k}}(\mathbf{r})+\int_{\text {u.c. }} \mathrm{d}^{3} \mathrm{r} \Psi_{n \mathbf{k}}^{*}(\mathbf{r}) \mathbf{r} \Psi_{n \mathbf{k}}(\mathbf{r}),
\end{aligned}
$$

where the integrals are performed over the unit cell.

Since the most interesting physical consequences of the Berry curvature occur in spin-orbit coupled systems, the theory is developed in terms of a fully relativistic multiple scattering theory based on the Dirac equation. In short, the four component Dirac Bloch wave [26] is expanded around the sites within the unit cell in terms of the local scattering solutions $\Phi_{Q}(\mathcal{E} ; \mathbf{r})$ of the radial Dirac equation as [19]

$$
\Psi_{n \mathbf{k}}(\mathbf{r})=\sum_{Q} C_{n, Q}(\mathbf{k}) \Phi_{Q}(\mathcal{E} ; \mathbf{r})
$$

where $Q$ comprises site and spin-angular momentum indices. In such a representation the expansion coefficients $C_{n, Q}(\mathbf{k})$ are solutions of the eigenvalue problem

$$
\overline{\bar{M}}(\mathcal{E} ; \mathbf{k}) \bar{C}_{n}(\mathbf{k})=\lambda_{n}(\mathcal{E} ; \mathbf{k}) \bar{C}_{n}(\mathbf{k}) \text { with } \bar{C}_{n}(\mathbf{k})=\left\{C_{n, Q}(\mathbf{k})\right\}
$$

for the KKR matrix [19]

$$
M_{Q Q^{\prime}}(\mathcal{E} ; \mathbf{k})=G_{Q Q^{\prime}}^{s}(\mathcal{E} ; \mathbf{k}) \Delta t_{Q^{\prime}}^{s}(\mathcal{E})-\delta_{Q Q^{\prime}}
$$

where $G_{Q Q^{\prime}}^{s}(\mathcal{E} ; \mathbf{k})$ are the relativistic screened structure constants, $\Delta t_{Q^{\prime}}^{s}(\mathcal{E})$ is the $\Delta t$ matrix of the reference system with respect to the system under consideration [24] and the eigenvalues $\lambda_{n}(\mathcal{E}, \mathbf{k})$ vanish for $\mathcal{E}=\mathcal{E}_{n \mathbf{k}}$.

Note, that the eigenvalue problem Eq. (49) depends parametrically on k. Therefore, one can formally deploy the original arguments of M. V. Berry [3] to derive an expression for the curvature associated with this problem. Namely, one finds (here for the sake of simplicity we assume the matrix $\overline{\bar{M}}$ to be Hermitian) [19]

$$
\mathbf{\Omega}_{n}^{\mathrm{KKR}}(\mathbf{k})=-\operatorname{Im}\left\{\sum_{m \neq n} \frac{\bar{C}_{n}^{\dagger} \nabla_{\mathbf{k}} \overline{\bar{M}} \bar{C}_{m} \times \bar{C}_{m}^{\dagger} \nabla_{\mathbf{k}} \overline{\bar{M}} \bar{C}_{n}}{\left(\lambda_{n}-\lambda_{m}\right)^{2}}\right\} .
$$

The r.h.s. has to be evaluated at an energy $\mathcal{E}=\mathcal{E}_{n \mathbf{k}}$, so $\lambda_{n}$ is equal to zero. Evidently, this curvature is a property of the KKR matrix $\overline{\bar{M}}(\mathcal{E} ; \mathbf{k})$. What is important about this result is the fact that the $\mathbf{k}$ derivative operates only on $\bar{M}(\mathcal{E} ; \mathbf{k})$ and therefore it can be expressed simply by

$$
\partial \overline{\bar{G}}^{s}(\mathcal{E} ; \mathbf{k}) / \partial \mathbf{k}=i \sum_{\mathbf{R}} \mathbf{R} e^{i \mathbf{k R}} \overline{\bar{G}}^{s}(\mathcal{E} ; \mathbf{R}),
$$

which can be easily evaluated since the screened real space structure constants $\overline{\bar{G}}^{s}(\mathcal{E} ; \mathbf{R})$ are short ranged. While this is reassuringly consistent with the expectation at the beginning of this Section it is not the whole story. The curvature of the KKR matrix is not the one of the Hamiltonian

$$
\hat{H}_{\mathbf{k}}(\mathbf{r})=e^{-i \mathbf{k} \cdot \mathbf{r}} \hat{H}(\mathbf{r}) e^{i \mathbf{k} \cdot \mathbf{r}}
$$

whose eigenfunctions are the periodic components $u_{n}(\mathbf{r}, \mathbf{k})$. In fact, when we evaluate Eq. (47) we find

$$
\mathbf{A}_{n}(\mathbf{k})=\mathbf{A}_{n}^{k}(\mathbf{k})+\mathbf{A}_{n}^{r}(\mathbf{k})=\mathbf{A}_{n}^{\mathrm{KKR}}(\mathbf{k})+\mathbf{A}_{n}^{v}(\mathbf{k})+\mathbf{A}_{n}^{r}(\mathbf{k})
$$


Here we have the KKR part

$$
\mathbf{A}_{n}^{\mathrm{KKR}}(\mathbf{k})=i \bar{C}_{n}^{\dagger} \boldsymbol{\nabla}_{\mathbf{k}} \bar{C}_{n}=-\operatorname{Im}\left\{\bar{C}_{n}^{\dagger} \nabla_{\mathbf{k}} \bar{C}_{n}\right\}
$$

the velocity part

$$
\mathbf{A}_{n}^{v}(\mathbf{k})=i \hbar \mathbf{v}_{n} \bar{C}_{n}^{\dagger} \overline{\bar{\Delta}} \bar{C}_{n}=-\hbar \mathbf{v}_{n} \operatorname{Im}\left\{\bar{C}_{n}^{\dagger} \overline{\bar{\Delta}} \bar{C}_{n}\right\}
$$

with

$$
(\overline{\bar{\Delta}})_{Q Q^{\prime}}(\mathcal{E})=\delta_{Q Q^{\prime}} \int_{\text {u.c. }} \mathrm{d}^{3} \mathrm{r} \Phi_{Q}^{\dagger}(\mathcal{E} ; \mathbf{r}) \frac{\partial \Phi_{Q^{\prime}}(\mathcal{E} ; \mathbf{r})}{\partial \mathcal{E}}
$$

and the dipole part

$$
\mathbf{A}_{n}^{r}(\mathbf{k})=\bar{C}_{n}^{\dagger}(\mathbf{k}) \overline{\overline{\mathbf{r}}} \bar{C}_{n}(\mathbf{k})
$$

with the matrix elements of the position operator

$$
(\overline{\overline{\mathbf{r}}})_{Q Q^{\prime}}(\mathcal{E})=\int_{\text {u.c. }} \mathrm{d}^{3} \mathrm{r} \Phi_{Q}^{\dagger}(\mathcal{E} ; \mathbf{r}) \mathbf{r} \Phi_{Q^{\prime}}(\mathcal{E} ; \mathbf{r})
$$

As will be shown, the contribution from the first term of r.h.s of Eq. (54) provides the curvature associated with the KKR matrix in Eq. (51), while the velocity term $\mathbf{A}_{n}^{v}(\mathbf{k})$ and dipole term $\mathbf{A}_{n}^{r}(\mathbf{k})$ lead to small corrections.

\subsubsection{Abelian Berry curvature}

Starting from the Berry connection introduced above it is straightforward to extend the method to the Berry curvature expressions. For the Abelian case the Berry curvature is given by three contributions

$$
\boldsymbol{\Omega}_{n}(\mathbf{k})=\nabla_{\mathbf{k}} \times \mathbf{A}_{n}(\mathbf{k})=\boldsymbol{\Omega}_{n}^{k}(\mathbf{k})+\boldsymbol{\Omega}_{n}^{r}(\mathbf{k})=\boldsymbol{\Omega}_{n}^{\mathrm{KKR}}(\mathbf{k})+\boldsymbol{\Omega}_{n}^{v}(\mathbf{k})+\boldsymbol{\Omega}_{n}^{r}(\mathbf{k})
$$

stemming from the analogue terms in Eq. (54). Here, the KKR part which is shown to be the dominant contribution [19] takes the simple form

$$
\mathbf{\Omega}_{n}^{\mathrm{KKR}}(\mathbf{k})=i \nabla_{\mathbf{k}} \bar{C}_{n}^{\dagger} \times \nabla_{\mathbf{k}} \bar{C}_{n}=-\operatorname{Im}\left\{\nabla_{\mathbf{k}} \bar{C}_{n}^{\dagger} \times \nabla_{\mathbf{k}} \bar{C}_{n}\right\}
$$

where just the expansion coefficients are involved. Now, an important step is to shift the $\mathbf{k}$ derivative towards the k-dependent KKR matrix. Actually, because of the chosen KKR-basis set in Eq. (48), one has to deal with a non-Hermitian KKR matrix. However, in order to simplify the further discussion and to have a clear insight into the presented approach, here the matrix $\overline{\bar{M}}$ is assumed to be Hermitian. Then, introducing a complete set of eigenvectors of the KKR matrix leads to Eq. (51) which is quite similar to the one obtained in the original paper of M. V. Berry [3]. The difference is just that the $\mathbf{k}$-dependent Hamiltonian is replaced by the $\mathbf{k}$-dependent KKR matrix of finite dimension. As was already discussed, the partial $\mathbf{k}$ derivative of the KKR matrix can be calculated analytically within the screened KKR method. In a similar way the velocity and the dipole terms, $\Omega_{n}^{v}(\mathbf{k})$ and $\Omega_{n}^{r}(\mathbf{k})$ can be treated. Both contributions are typically one order of magnitude smaller than the KKR part [19]. Moreover, the velocity part of the Berry curvature is always strictly perpendicular to the group velocity. This fact is a technically very important feature since for a Fermi surface integral over the Berry curvature this contribution vanishes. 

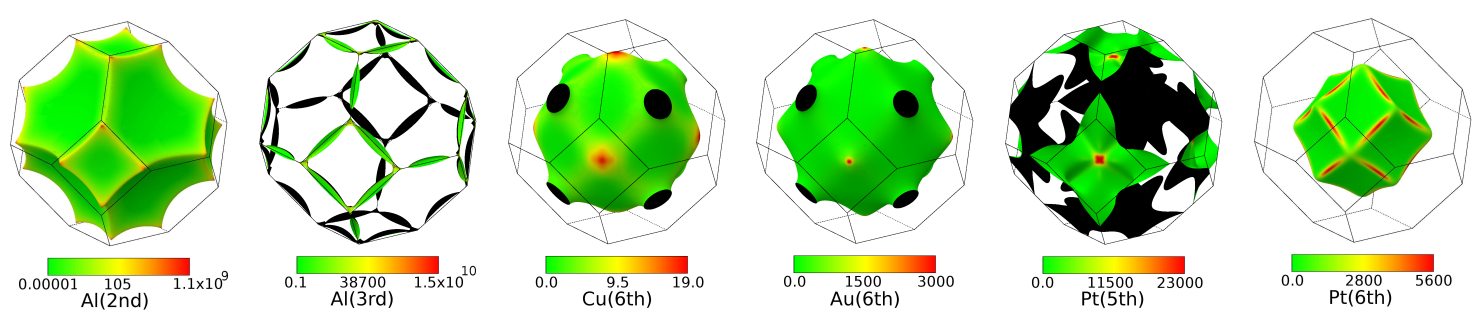

Figure 7: The absolute value (in a.u.) of the determinant of the non-Abelian Berry curvature $|\operatorname{Det}| \boldsymbol{\Omega}_{i j}(\mathbf{k})||$ for the Fermi surface of several metals. From left to right Al (2nd and 3rd band), $\mathrm{Cu}$ (6th band), Au (6th band) and Pt (5th and 6th band). For Al we used a logarithmic scale to pronounce the important regions.

\subsubsection{Non-Abelian Berry curvature}

For a treatment of the non-Abelian Berry curvature not only the conventional connection has to be taken into account, but also the commutator, which is provided by the requirement of the gauge invariant theory, has to be considered [5]. As a consequence, the Berry curvature is defined as

$$
\boldsymbol{\Omega}_{i j}(\mathbf{k})=i\left\langle\boldsymbol{\nabla}_{\mathbf{k}} u_{i}(\mathbf{k})|\times| \nabla_{\mathbf{k}} u_{j}(\mathbf{k})\right\rangle-i \sum_{l \in \Sigma}\left\langle\nabla_{\mathbf{k}} u_{i}(\mathbf{k}) \mid u_{l}(\mathbf{k})\right\rangle \times\left\langle u_{l}(\mathbf{k}) \mid \nabla_{\mathbf{k}} u_{j}(\mathbf{k})\right\rangle .
$$

Here, as has been discussed in section $1.3, \Sigma$ contains all indices of the degenerate subspace. Rewriting it in terms of the Bloch states yields

$$
\begin{aligned}
\boldsymbol{\Omega}_{i j}(\mathbf{k}) & =i\left\langle\nabla_{\mathbf{k}} \Psi_{i \mathbf{k}}|\times| \nabla_{\mathbf{k}} \Psi_{j \mathbf{k}}\right\rangle+\left\langle\nabla_{\mathbf{k}} \Psi_{i \mathbf{k}} \times \mathbf{r} \mid \Psi_{j \mathbf{k}}\right\rangle-\left\langle\Psi_{i \mathbf{k}} \mid \mathbf{r} \times \nabla_{\mathbf{k}} \Psi_{j \mathbf{k}}\right\rangle- \\
& -\sum_{l \in \Sigma}\left\{i\left\langle\nabla_{\mathbf{k}} \Psi_{i \mathbf{k}} \mid \Psi_{l \mathbf{k}}\right\rangle \times\left\langle\Psi_{l \mathbf{k}} \mid \nabla_{\mathbf{k}} \Psi_{j \mathbf{k}}\right\rangle-\left\langle\Psi_{i \mathbf{k}}|\mathbf{r}| \Psi_{l \mathbf{k}}\right\rangle \times\left\langle\Psi_{l \mathbf{k}} \mid \nabla_{\mathbf{k}} \Psi_{j \mathbf{k}}\right\rangle+\right. \\
& \left.+\left\langle\nabla_{\mathbf{k}} \Psi_{i \mathbf{k}} \mid \Psi_{l \mathbf{k}}\right\rangle \times\left\langle\Psi_{l \mathbf{k}}|\mathbf{r}| \Psi_{j \mathbf{k}}\right\rangle+i\left\langle\Psi_{i \mathbf{k}}|\mathbf{r}| \Psi_{l \mathbf{k}}\right\rangle \times\left\langle\Psi_{l \mathbf{k}}|\mathbf{r}| \Psi_{j \mathbf{k}}\right\rangle\right\}
\end{aligned}
$$

and a similar decomposition into the KKR, velocity and dipole parts

$$
\boldsymbol{\Omega}_{i j}(\mathbf{k})=\boldsymbol{\Omega}_{i j}^{\mathrm{KKR}}(\mathbf{k})+\boldsymbol{\Omega}_{i j}^{v}(\mathbf{k})+\boldsymbol{\Omega}_{i j}^{r}(\mathbf{k})
$$

can be performed.

In Fig. 7 the absolute value of the determinant $|\operatorname{Det}| \Omega_{i j}^{z}(\mathbf{k})||$ of the non-Abelian (vector-valued matrix) Berry curvature over the Fermi surface of several metals is shown. This quantity is gauge invariant as has been discussed in section 1.5) and is convenient to be visualized for a Kramers degenerate band. Near degeneracies at the Fermi level lead to enhanced Berry curvatures as can be seen for $\mathrm{Al}$ and $\mathrm{Pt}$. Interestingly, the values for $\mathrm{Al}$ exceeds much larger values than in $\mathrm{Au}$ even it is much lighter than the noble metal. The origin are the mentioned near degeneracies of $\mathrm{Al}$ at the Fermi level which were discussed in the literature [22, 20]. Furthermore, the energy resolved Berry curvature (here $S(\mathcal{E})$ is the isosurface of constant energy $\mathcal{E}$ in the k space)

$$
\Omega^{z}(\mathcal{E})=\sum_{n} \frac{1}{\hbar} \int_{S(\mathcal{E})} \frac{d^{2} k}{\left|v^{n}(\mathbf{k})\right|} \Omega_{n}^{z, \uparrow}(\mathbf{k})
$$


is shown in Fig. 8. Here $\Omega_{n}^{z, \uparrow}(\mathbf{k})$ denotes the Berry curvature of the degenerate band with the positive spin polarization $\left\langle\Psi_{\uparrow \mathbf{k}}\left|\beta \Sigma_{z}\right| \Psi_{\uparrow \mathbf{k}}\right\rangle>0$. It proves that the KKR part of Eq. (64) is the dominant contribution to the Berry curvature. Clearly visible is the quite spiky structure of the curvature as a function of the energy. As discussed in the literature, this makes the integration of the Berry curvature computationally demanding, requiring a large number of $\mathbf{k}$ and energy points.

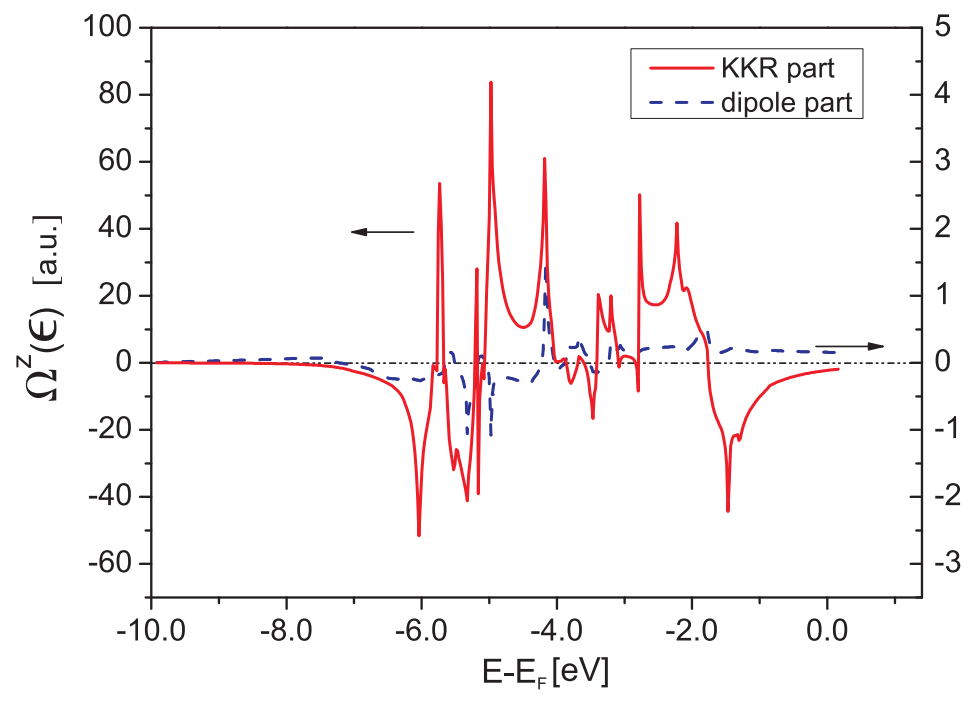

Figure 8: The energy resolved Berry curvature $\Omega^{z}(\mathcal{E})$ for Au divided into the parts $\Omega^{\mathrm{KKR}}(\mathcal{E})$ and $\Omega^{r}(\mathcal{E})$ according to Eq. (64) and Ref. [19]. The part $\Omega^{v}(\mathcal{E})$ is negligibly small and not shown.

\section{$2.2 \quad$ Tight-Binding Model}

The tight-binding (TB) model provides a convenient framework for studying the geometric quantities in band theory. We will briefly introduce the tight-binding method with spin-orbit coupling and exchange interaction. The usefulness of this model is then illustrated by discussing interesting features of the Berry curvature of a simple band structure, studied in Ref. [11].

\subsubsection{Definition of the TB-Hamiltonian}

The tight-binding model assumes that the electronic wave function at each lattice site is well localized around the position of the atom. For this purpose one assumes the crystal potential to consist of a sum of spherically symmetric, somewhat strongly attractive potentials located at the core positions. The idea is to treat the overlap matrix elements as a perturbation and consequently expand the wave function in a basis of atomic orbitals $\left\{\phi_{\alpha}(\mathbf{r}-\mathbf{R})\right\}$. The ansatz

$$
\Psi_{n \mathbf{k}}(\mathbf{r})=\frac{1}{\sqrt{N_{R}}} \sum_{\mathbf{R}} e^{i \mathbf{k R}} \sum_{\alpha} C_{n \alpha}(\mathbf{k}) \phi_{\alpha}(\mathbf{r}-\mathbf{R})
$$

ensures that $\Psi_{n \mathbf{k}}$ fulfills the Bloch theorem with the lattice vectors $\mathbf{R}$. The simplest case involves neglecting the overlap matrix elements of the wave function

$$
\left\langle\phi_{\beta}(\mathbf{r}-\mathbf{R}) \mid \phi_{\alpha}\left(\mathbf{r}-\mathbf{R}^{\prime}\right)\right\rangle \sim \delta_{\alpha, \beta} \delta_{\mathbf{R}, \mathbf{R}^{\prime}}
$$


The normalized eigenfunctions in this on-site approximation are subject to the eigenvalue problem $\left\langle\Psi_{n \mathbf{k}}|H| \Psi_{n \mathbf{k}}\right\rangle=\mathcal{E}_{n \mathbf{k}}$. The solution to this problem in the tight-binding formulation of Eq. (66) requires the diagonalization of the tight-binding matrix

$$
H_{\alpha \beta}(\mathbf{k})=\sum_{\mathbf{R}, \mathbf{R}^{\prime}} e^{i \mathbf{k}\left(\mathbf{R}-\mathbf{R}^{\prime}\right)}\left\langle\phi_{\alpha}\left(\mathbf{r}-\mathbf{R}^{\prime}\right)|H| \phi_{\beta}(\mathbf{r}-\mathbf{R})\right\rangle .
$$

Then the coefficients $C_{n \alpha}(\mathbf{k})$ are obtained as the components of the eigenvector of this matrix and thus the eigenstates necessary for the evaluation of the Berry curvature are easily accessible. The matrix elements in Eq. (68) can be parametrized by the method of Slater and Koster [27]. In order to observe a nontrivial Berry curvature we need to take into account spin degrees of freedom. This doubles the number of bands compared to the spinless case. Within the scope of the tight-binding model the spin-orbit interaction is treated as a perturbation

$$
V_{\mathrm{SOC}}=\frac{\hbar}{4 m^{2} c^{2}}\left(\nabla_{\mathbf{r}} V(\mathbf{r}) \times \mathbf{p}\right) \mathbf{S}=V_{\mathrm{SOC}}=\frac{2 \lambda}{\hbar^{2}} \mathbf{L S}
$$

to the Hamiltonian. Here a spherically symmetric potential is assumed and the parameter $\lambda$ is considered to be small with respect to the on-site and hopping energies in Eq. (68). The matrix elements of this operator have been listed elsewhere for basis states of $p, d$ and $f$ symmetry [28] in on-site approximation, although the formalism presented here does not require this approximation.

Furthermore, one can use this model to describe a ferromagnetic material by incorporating an exchange interaction term. The simplest formulation originates from the mean field theory. Without taking into account any temperature dependence, a constant exchange field is assumed and the $z$ axis is chosen as the quantization direction

$$
H_{\mathrm{xc}}=-\mathbf{V}_{\mathrm{xc}} \boldsymbol{\sigma}=-V_{\mathrm{xc}} \sigma_{z}
$$

where $V_{\mathrm{xc}}$ is a positive real number.

\subsubsection{Berry Curvature}

As in the case of the KKR method discussed in the previous Section, solving the eigenproblem of the tight-binding matrix gives the Bloch wave $\left|\Psi_{n \mathbf{k}}\right\rangle$ instead of the periodic function $\left|u_{n}(\mathbf{k})\right\rangle$. Hence, one also needs to consider the two parts, $\boldsymbol{\Omega}^{k}(\mathbf{k})$ and $\boldsymbol{\Omega}^{r}(\mathbf{k})$, of the Berry curvature introduced by Eq. (60). Exploiting the on-site approximation of Eq. (67) and the normalization condition for the coefficents, one gets the first term for the Abelian case in a well known form

$$
\begin{aligned}
\boldsymbol{\Omega}_{n}^{k}(\mathbf{k}) & =i\left\langle\nabla_{\mathbf{k}} \Psi_{n \mathbf{k}}|\times| \nabla_{\mathbf{k}} \Psi_{n \mathbf{k}}\right\rangle_{\text {u.c. }}=i \nabla_{\mathbf{k}} \bar{C}_{n}^{\dagger}(\mathbf{k}) \times \nabla_{\mathbf{k}} \bar{C}_{n}(\mathbf{k}) \\
& =-\operatorname{Im} \sum_{m \neq n} \frac{\bar{C}_{n}^{\dagger}(\mathbf{k}) \nabla_{\mathbf{k}} \overline{\bar{H}}(\mathbf{k}) \bar{C}_{m}(\mathbf{k}) \times \bar{C}_{m}^{\dagger}(\mathbf{k}) \nabla_{\mathbf{k}} \overline{\bar{H}}(\mathbf{k}) \bar{C}_{n}(\mathbf{k})}{\left(\mathcal{E}_{n \mathbf{k}}-\mathcal{E}_{m \mathbf{k}}\right)^{2}} .
\end{aligned}
$$

The second, the dipole term, is given by

$$
\begin{aligned}
\boldsymbol{\Omega}_{n}^{r}(\mathbf{k}) & =\nabla_{\mathbf{k}} \times\left\langle\Psi_{n \mathbf{k}}|\mathbf{r}| \Psi_{n \mathbf{k}}\right\rangle_{\text {cell }}=\nabla_{\mathbf{k}} \times\left(\bar{C}_{n}^{\dagger}(\mathbf{k}) \overline{\overline{\mathbf{r}}} \bar{C}_{n}(\mathbf{k})\right) \\
& =\sum_{m \neq n} 2 \operatorname{Re}\left[\frac{\bar{C}_{n}^{\dagger}(\mathbf{k}) \nabla_{\mathbf{k}} \overline{\bar{H}}(\mathbf{k}) \bar{C}_{m}(\mathbf{k})}{\mathcal{E}_{n \mathbf{k}}-\mathcal{E}_{m \mathbf{k}}} \times\left(\bar{C}_{m}^{\dagger}(\mathbf{k}) \overline{\overline{\mathbf{r}}} \bar{C}_{n}(\mathbf{k})\right)\right]
\end{aligned}
$$


where we have introduced the vector valued matrix $\overline{\overline{\mathbf{r}}}$ with the components $\mathbf{r}_{\alpha \beta}=\left\langle\phi_{\beta}(\mathbf{r})|\mathbf{r}| \phi_{\alpha}(\mathbf{r})\right\rangle$. Similar to the screened KKR method, the $\mathbf{k}$ derivative of $\overline{\bar{H}}(\mathbf{k})$ in the equations above may be performed analytically and no numerical derivative is needed.

In the case of degenerate bands, the non-Abelian Berry curvature $\boldsymbol{\Omega}_{i j}(\mathbf{k})=\boldsymbol{\Omega}_{i j}^{k}(\mathbf{k})+\boldsymbol{\Omega}_{i j}^{r}(\mathbf{k})$ is expressed in the following terms (according to section 1.3, $\Sigma$ contains all indices of the degenerate subspace)

$$
\begin{aligned}
\boldsymbol{\Omega}_{i j}^{k}(\mathbf{k})= & i \sum_{m \notin \Sigma} \frac{\bar{C}_{i}^{\dagger}(\mathbf{k}) \nabla_{\mathbf{k}} \overline{\bar{H}}(\mathbf{k}) \bar{C}_{m}(\mathbf{k}) \times \bar{C}_{m}^{\dagger}(\mathbf{k}) \nabla_{\mathbf{k}} \overline{\bar{H}}(\mathbf{k}) \bar{C}_{j}(\mathbf{k})}{\left(\mathcal{E}_{i \mathbf{k}}-\mathcal{E}_{m \mathbf{k}}\right)\left(\mathcal{E}_{j \mathbf{k}}-\mathcal{E}_{m \mathbf{k}}\right)} \\
\boldsymbol{\Omega}_{i j}^{r}(\mathbf{k})= & \sum_{m \notin \Sigma}\left[-\left(\bar{C}_{i}^{\dagger}(\mathbf{k}) \overline{\mathbf{r}} \bar{C}_{m}(\mathbf{k})\right) \times \frac{\bar{C}_{m}^{\dagger}(\mathbf{k}) \nabla_{\mathbf{k}} \overline{\bar{H}}(\mathbf{k}) \bar{C}_{j}(\mathbf{k})}{\mathcal{E}_{j \mathbf{k}}-\mathcal{E}_{m \mathbf{k}}}\right. \\
& \left.+\frac{\bar{C}_{i}^{\dagger}(\mathbf{k}) \nabla_{\mathbf{k}} \overline{\bar{H}}(\mathbf{k}) \bar{C}_{m}(\mathbf{k})}{\mathcal{E}_{i \mathbf{k}}-\mathcal{E}_{m \mathbf{k}}} \times\left(\bar{C}_{m}^{\dagger}(\mathbf{k}) \overline{\mathbf{r}} \bar{C}_{j}(\mathbf{k})\right)\right] \\
& -i \sum_{l \in \Sigma}\left(\bar{C}_{i}^{\dagger}(\mathbf{k}) \overline{\mathbf{r}} \bar{C}_{l}(\mathbf{k})\right) \times\left(\bar{C}_{l}^{\dagger}(\mathbf{k}) \overline{\overline{\mathbf{r}}} \bar{C}_{j}(\mathbf{k})\right)
\end{aligned}
$$

Here only the last term is not a direct generalization of the Abelian Berry curvature. Again it is possible to circumvent the numerical derivative by a summation over all states that do not belong to the degenerate subspace. The last term does not involve any derivative, therefore the sum runs only over the degenerate bands.

\subsubsection{Diabolical Points}

To illustrate the behavior of the Berry curvature near degeneracies, we present calculations of a simple band structure using the tight-binding method. We consider a ferromagnetic simple cubic crystal with eight bands including one band with $s$ symmetry and three bands with $p$ symmetry for each spin direction. Due to the exchange interaction there is no time-reversal symmetry and the codimension of degeneracies is three.

Regions of the parameter space with a higher symmetry (e.g., high symmetry lines in the Brillouin zone) are more likely to support accidental degeneracies because the symmetry possibly reduces the codimension only in this region. Level crossings on a high symmetry line in the Brillouin zone do not necessarily occur on account of symmetry as long as the bands are not degenerate at points nearby which have the same symmetry.

As an example, in Fig. 9 a few bands of the band structure near the $\Gamma$ point along a high symmetry line are plotted. There occur three crossings between different bands. On the righthand side, the energy dispersion in a plane through the degeneracy marked by $\mathrm{P}$ is displayed in order to show the cone shape of the intersection. The color scale represents the spin polarization $\left\langle\Psi\left|\sigma_{z}\right| \Psi\right\rangle$ of the corresponding band. Within one band the spin polarization rapidly changes in the vicinity of the degeneracy. At the degeneracy itself it jumps due to the cusp of the corresponding band. However, when passing the intersection along a straight line in $\mathbf{k}$ space and jumping from the lower to the upper band apparently the spin polarization does not change at all. This is a clear indication that the character of the two bands is exchanged at the intersection.

The behavior of the spin polarization near the diabolical point illustrates how observables are 

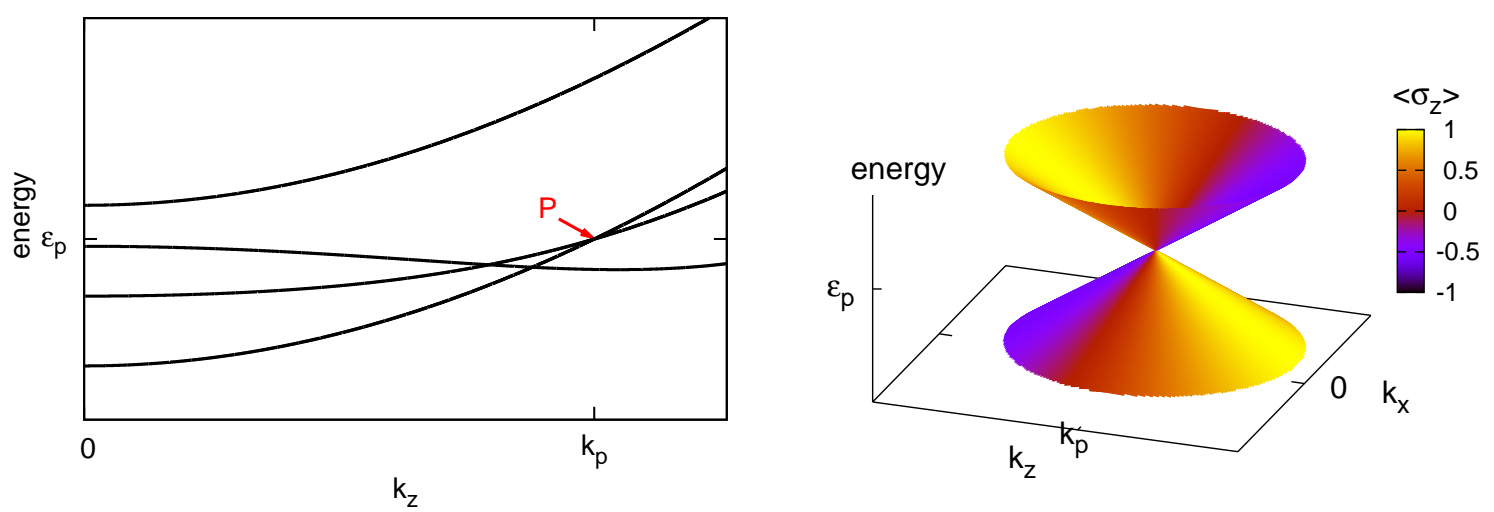

Figure 9: Left: The crossing of some $p$ bands of a ferromagnetic simple cubic band structure near the center of the Brillouin zone. Right: Conical intersection of energy surfaces near point $\mathrm{P}$. The $z$ axis represents the energy dispersion of two intersecting bands over an arbitrary plane in $\mathbf{k}$ space through the diabolical point. The color scale denotes the spin polarization corresponding to each band.

influenced by other bands nearby. The Berry curvature can be viewed as a measure of this coupling which becomes evident from Eqs. (71) and (72), where a sum over all other bands is performed weighted by the inverse energy difference. Hence, the Berry curvature of a single Bloch band generally arises due to the restriction to this band and it becomes large when other bands are close. As we have seen before, a degeneracy produces a singularity of the Berry curvature and the adiabatic single-band approximation fails.

In our case the origin of the Berry curvature is the spin-orbit coupling. Since degenerate or almost degenerate points in the band structure produce large spin mixing of the involved bands, a peak in the Berry curvature can also be understood from this point of view.

As discussed in Section 1.4, we would expect the curvature around the degeneracy to obey the $1 /\left|\mathbf{k}-\mathbf{k}^{*}\right|^{2}$-law of a Coulomb field. The asymptotic behavior becomes evident when plotting $1 / \sqrt{|\boldsymbol{\Omega}|}$ in a plane the degeneracy is located in(see Fig. 10). We observe an absolute value function $\left|\mathbf{k}-\mathbf{k}^{*}\right|$, which proves that the Berry curvature really has the form of the monopole field strength given by Eq. (40).

Besides the absolute value we can also examine the direction of the Berry curvature vector. In
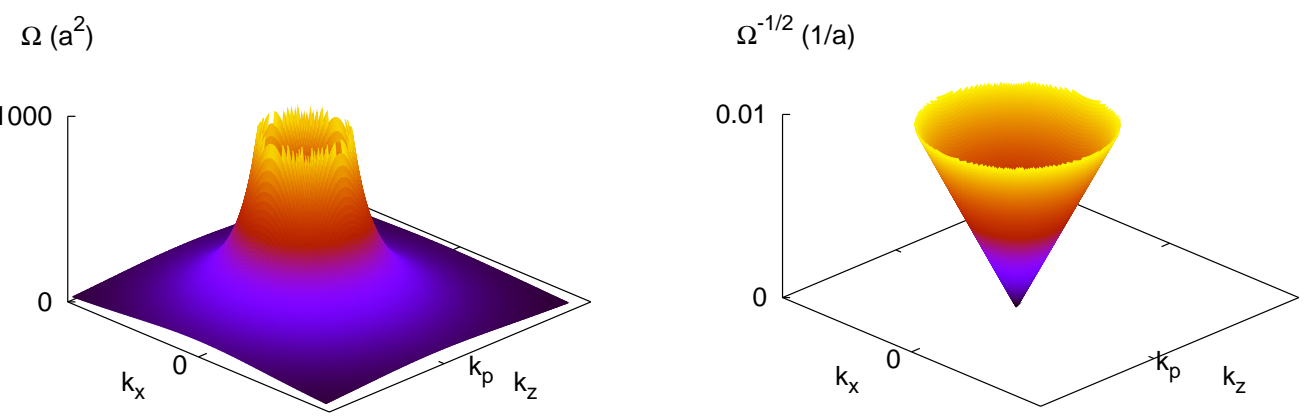

Figure 10: Left: Absolute value of the Berry curvature $|\boldsymbol{\Omega}|$ plotted over a plane in $\mathbf{k}$ space through the degeneracy point P. Right: Inverse square root of the absolute value, i.e., $1 / \sqrt{|\boldsymbol{\Omega}|}$. 
Fig. 11, we recognize the characteristic monopole field. In the lower band the monopole is a source, in the upper band a drain of the Berry curvature. This has been expected because a monopole in one band has to be matched by a monopole of opposite charge in the other band involved in the degeneracy.
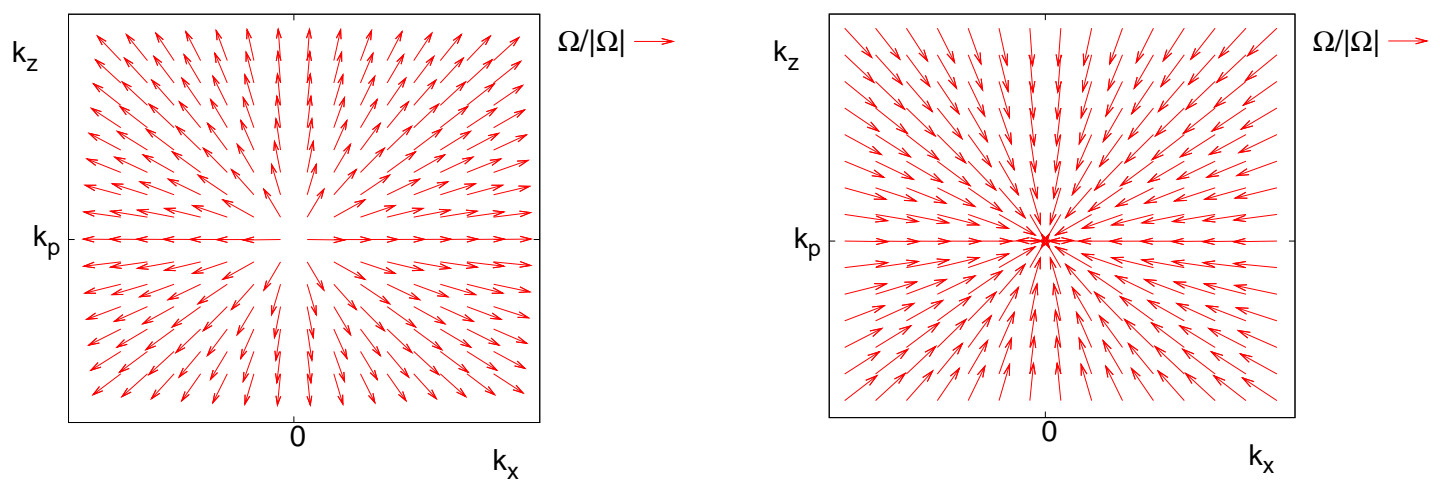

Figure 11: Normalized Berry curvature vector around $P$ (see Fig. 9).

In general, we may assign a "charge" $g$ to the monopole as in Eq. (42). This charge is quantized to be either integer or half integer. In order to determine the charge numerically one could perform a fit of the numerical data with the monopole field strength. However, there is no reason to believe that all directions in $\mathbf{k}$ space must be equivalent. Some distortion in a certain direction might result in ellipsoidal isosurfaces of $|\boldsymbol{\Omega}|$ instead of spherical ones. A different approach, independent from the coordinate system exploits Eq. (41)

$$
\int_{V} \mathrm{~d}^{3} \mathbf{k} \boldsymbol{\nabla}_{\mathbf{k}} \cdot \boldsymbol{\Omega}_{n}(\mathbf{k})=\int_{\partial V} \mathrm{~d} s \mathbf{n} \cdot \boldsymbol{\Omega}_{n}(\mathbf{k})=2 \pi m \quad m \in \mathbb{Z},
$$

where the vector $\mathbf{n}$ is normal on the bounding surface $\partial V$ of some arbitrary volume $V$. Performing the numerical surface integration causes no problem since the Berry curvature is analytical on the surface unlike at the degeneracy.

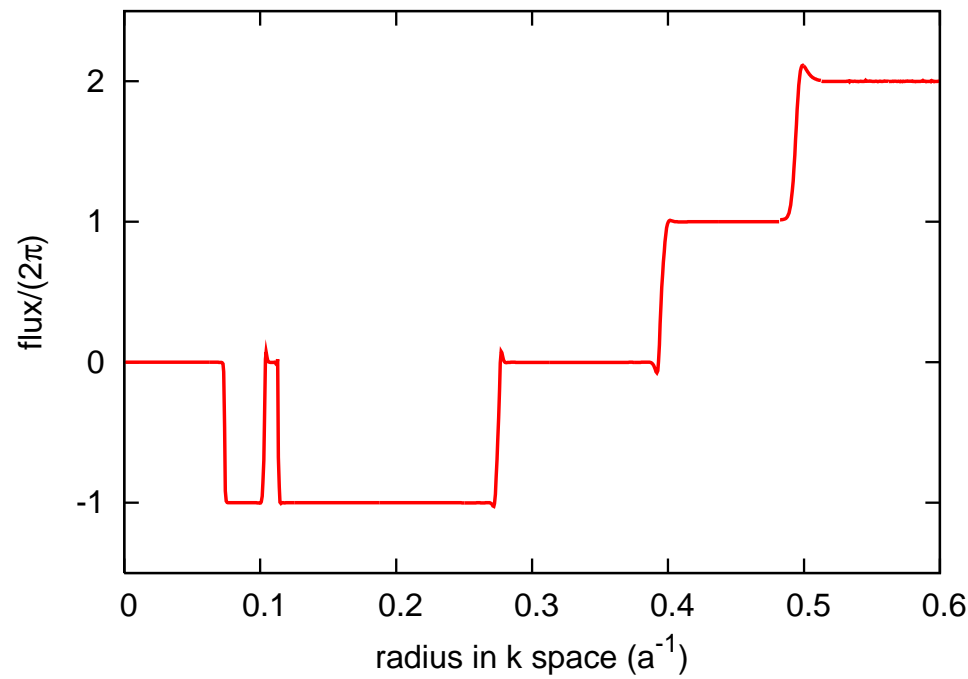

Figure 12: The value of the integral Eq. (75) over a spherical surface for the upper band is plotted against the radius of the sphere.

So as to validate this formula, the Berry curvature flux through a sphere of radius $\left|\mathbf{k}-\mathbf{k}_{0}\right|$ 
centered at a generic point $\mathbf{k}_{0}$ in the Brillouin zone near $P$ (see Fig. 9) is evaluated. This flux divided by $2 \pi$ as a function of the sphere's radius is plotted in Fig. 12 .

The integral is observed to be quantized to integer multiples of $2 \pi$. When increasing the radius of the sphere the surface crosses various diabolical points. Each time this happens the flux jumps by $\pm 2 \pi$ depending on whether the charge of the Berry curvature monopole is positive or negative. According to Eq. (75), this confirms that the charges of the monopoles created by the point-like degeneracies are $g= \pm 1 / 2$. Altogether there are six jumps, which means there are six diabolical points in the vicinity of the point $P$ (see Fig. 9 ). Two of the monopoles in the upper band have a negative, the other four a positive charge of $1 / 2$. A generic point as the center of the integration sphere is chosen to avoid crossing more than one diabolical point at the time. The deviations from a perfect step function are due to the discretization of the integral, which increases the error when the surface crosses a monopole. The step functions also verify the statement that monopoles are the only possible sources of the Berry curvature.

F. D. M. Haldane [16] describes the dynamics of these degeneracies with respect to the variation of some control parameter, including the creation of a pair of diabolical points and their recombination after relative displacement of a reciprocal lattice vector. In the considered case an obvious choice for the control parameters would be $\lambda$ or $V_{\mathrm{xc}}$, regulating the strength of spin-orbit coupling or exchange splitting, respectively.

Thus, the tight-binding code provides an excellent tool for an investigation of the effects connected with the accidental degeneracy of bands (see for instance, Ref. [16]) since one may scan through a whole range of parameters without consuming much computational resources and still obtain qualitatively reliable results.

\subsection{Wannier Interpolation scheme}

The first method developed specifically for calculating the Berry curvature of Bloch electrons using the full machinery of the Density Functional Theory (DFT) was based on the Wannier interpolation code of Marzari, Souza and Vanderbilt [29]. As in the case of the KKR method presented in Section 2.1, the essence of this approach by Wang et al. [21] is that it avoids taking the derivative of the periodic part of the Bloch function $u_{n}(\mathbf{r}, \mathbf{k})$ with respect to $\mathbf{k}$ numerically by finite differences. A second almost equally important feature is that it offers various opportunities to interpolate between different $\mathbf{k}$ points in the Brillouin zone and hence reduces the number of points at which full ab initio calculations need to be performed.

Note that the formulas, naturally arising in wave packet dynamics, for the Berry connection and curvature, given by Eqs. (20) and (21), involve the real space integrals over a unit cell only. Extending them to cover all space by using the Wannier functions defined as

$$
|\mathbf{R} n\rangle=\frac{V_{u . c .}}{(2 \pi)^{3}} \int_{B Z} \mathrm{~d}^{3} \mathrm{k} e^{-i \mathbf{k} \cdot \mathbf{R}}\left|\Psi_{n \mathbf{k}}\right\rangle,
$$

the Bloch theorem and some care with the algebra one finds the following remarkably simple results $[21,30]$

$$
\mathbf{A}_{n}(\mathbf{k})=\sum_{\mathbf{R}}\langle 0 n|\mathbf{r}| \mathbf{R} n\rangle e^{i \mathbf{k} \cdot \mathbf{R}} \quad \text { and } \quad \boldsymbol{\Omega}_{n}(\mathbf{k})=i \sum_{\mathbf{R}}\langle 0 n|\mathbf{R} \times \mathbf{r}| \mathbf{R} n\rangle e^{i \mathbf{k} \cdot \mathbf{R}} .
$$


wThe matrix elements with respect to the Wannier states, as usual, involve integrals over all space. These formulas turn up and play a central role in the method of Wang et al. [21] and make the Wannier interpolation approach looking different from that based on the KKR method which makes use of expansions and integration within a single unit cell only. The lattice sums in Eq. (77) do not have convenient convergence properties, as they depend on the tails of the Wannier functions in real space. Important to note is the fact that the phase freedom of the Bloch states allows for an optimization of these tails to reduce the numerical effort. By the time an efficient procedure giving "maximally localized" Wannier functions [29, 30] is developed to deal with this issue, where also matrix elements of both $\boldsymbol{\nabla}_{\mathbf{k}}$ and $\mathbf{r}$, as in the KKR method discussed in Section (2.1), occur. In fact, reassuringly, it is found in both approaches that an easy to evaluate matrix element of $\boldsymbol{\nabla}_{\mathbf{k}}$ dominates over what is frequently called the dipole contribution involving matrix elements of $\mathbf{r}$. The Wannier orbitals are localized, but unlike the orbitals in the tight-binding method, exact representations of the band structure of a periodic solid within this method is possible only for a limited energy range. For metals, its use in modern first-principle calculations is based on the unique "maximally localized orbitals" and its power and achievements are well summarized in Ref. [29]. Here we wish to recall only the bare outline of the new development occasioned by the current interest in the geometrical and topological features of the electronic structure of crystals.

The method based on the Wannier interpolation scheme, well described in Ref. [21], starts with a conventional DFT calculation of Bloch states $\left|\Psi_{n \mathbf{q}}\right\rangle$ in a certain energy range and on a selected mesh of $\mathbf{q}$ points based on plane wave expansion. Then the matrix elements of various operators may be constructed with respect to a set of maximally localized Wannier states $|\mathbf{R} n\rangle$. It should be mention that generally those states are distinct from the Wannier states defined in Eq. (76) since they are solutions of a procedure to minimize the real space spread of the Wannier functions [30]. The minimization is always possible due to the freedom choosing an arbitrary phase in the definition of Bloch states as mentioned above. In general, this freedom in defining the Bloch states can be written in terms of a unitary operator. If we assume a set of Wannier states was chosen according to this procedure the phase sum of these states

$$
\left|u_{n}^{W}(\mathbf{k})\right\rangle=\sum_{\mathbf{R}} e^{-i \mathbf{k} \cdot(\mathbf{r}-\mathbf{R})}|\mathbf{R} n\rangle
$$

can be defined for an arbitrary $\mathbf{k}$ point, which may be at or in between the first principles q-point mesh. It can be regarded as a "Wannier gauge" representation of the periodic part of a Bloch state but they are generally no eigenstates of the $\mathbf{k}$ dependent Hamiltonian. In the following one has to evaluate the matrix elements with respect to the constructed states

$$
\begin{aligned}
H_{n m}^{W}(\mathbf{k}) & =\sum_{\mathbf{R}} e^{+i \mathbf{k} \cdot \mathbf{R}}\langle\mathbf{0} n|\widehat{H}| \mathbf{R} m\rangle, \\
\boldsymbol{\nabla} H_{n m}^{W}(\mathbf{k}) & =\sum_{\mathbf{R}} e^{+i \mathbf{k} \cdot \mathbf{R}} i \mathbf{R}\langle\mathbf{0} n|\widehat{H}| \mathbf{R} m\rangle, \\
\mathbf{A}_{n m}^{W}(\mathbf{k}) & =\sum_{\mathbf{R}} e^{+i \mathbf{k} \cdot \mathbf{R}}\langle\mathbf{0} n|\mathbf{r}| \mathbf{R} m\rangle, \\
\mathbf{\Omega}_{n m}^{W}(\mathbf{k}) & =i \sum_{\mathbf{R}} e^{+i \mathbf{k} \cdot \mathbf{R}}\langle\mathbf{0} n|\mathbf{R} \times \mathbf{r}| \mathbf{R} m\rangle .
\end{aligned}
$$

Actually, all of them are given in the same "Wannier gauge". The indices $n$ and $m$ refer to the full bundle of Wannier states selected to represent the real bands up to a certain energy above 
the Fermi level.

The next step is to find a unitary matrix $\overline{\bar{U}}(\mathbf{k})$ such that

$$
\overline{\bar{U}}^{\dagger}(\mathbf{k}) \overline{\bar{H}}^{W}(\mathbf{k}) \overline{\bar{U}}(\mathbf{k})=\overline{\bar{H}}^{H}(\mathbf{k}) \text { with } H_{n m}^{H}(\mathbf{k})=\mathcal{E}_{n \mathbf{k}} \delta_{n m}
$$

where the eigenvalue $\mathcal{E}_{n \mathbf{k}}$ should agree with the first-principles dispersion relation of the bands chosen to be represented. The corresponding eigenfunctions

$$
\left|u_{n}^{H}(\mathbf{k})\right\rangle=\sum_{m} U_{n m}(\mathbf{k})\left|u_{m}^{W}(\mathbf{k})\right\rangle
$$

should reproduce the periodic part of these states.

Thus, $\left|u_{n}^{H}(\mathbf{k})\right\rangle$ can be used to evaluate Eqs. (20) and (21) for the Berry connection and the curvature in the standard way. However, such direct calculations are precisely not what one would like to do. The point of Wang et al. is that the above preamble offers an alternative. Namely, the unitary transformation $\overline{\bar{U}}(\mathbf{k})$ transforms all states and operators from the "Wannier gauge" to another gauge which is called "Hamiltonian $(\mathrm{H})$ gauge" and one can transform all the easy to evaluate "Wannier gauge" operators in Eq. (79) into their "Hamiltonian gauge" form. Of course, $\boldsymbol{\Omega}_{n m}^{H}(\mathbf{k})$ is of particular interest. Unfortunately, due to the $\mathbf{k}$ dependence of $U_{n m}(\mathbf{k})$ the form of Eqs. (20) and (21) is not covariant under such a transformation. For instance the connection is given by

$$
\mathbf{A}^{H}=U^{\dagger} \mathbf{A}^{W} U+i U^{\dagger} \nabla U=\overline{\mathbf{A}}^{H}+i U^{\dagger} \nabla U
$$

where for clarity the momentum dependency has been dropped as we will mainly do within this section. All products are matrix-matrix multiplications and the gradient is taken with respect to k. A similar, but more complicated formula can be derived for $\Omega^{H}$. The matrices $\overline{\mathbf{A}}^{H}, \overline{\mathbf{A}}^{H}$, and $\bar{H}^{H}$ are denoted as the covariant components, that is to say the part of the transformed operator which does not contain $U^{\dagger} \boldsymbol{\nabla} U$, of $\mathbf{A}^{H}$ and $H^{H}$, respectively. If we write $\overline{\boldsymbol{\Omega}}^{H}=U^{\dagger} \boldsymbol{\Omega}^{W} U$ and

$$
\mathbf{D}_{n m}^{H}=\left(U^{\dagger} \nabla U\right)_{n m}=\frac{\left(U^{\dagger} \nabla \bar{H}^{W} U\right)_{n m}}{\mathcal{E}_{m}-\mathcal{E}_{n}}\left(1-\delta_{n m}\right),
$$

the final formula for the total Berry curvature $\boldsymbol{\Omega}(\mathbf{k})=\sum_{n} f_{n} \boldsymbol{\Omega}_{n}(\mathbf{k})$, including the sum over all occupied states, reads as follows

$$
\boldsymbol{\Omega}(\mathbf{k})=\sum_{n} f_{n} \overline{\mathbf{\Omega}}_{n n}^{H}+\sum_{n, m}\left(f_{m}-f_{n}\right) \mathbf{D}_{n m}^{H} \times \overline{\mathbf{A}}_{m n}^{H}+\mathbf{\Omega}^{D D}
$$

where the last term takes the following form

$$
\mathbf{\Omega}^{D D}=i \sum_{n, m}\left(f_{m}-f_{n}\right) \frac{\left(U^{\dagger} \nabla \bar{H}^{W} U\right)_{n m} \times\left(U^{\dagger} \nabla \bar{H}^{W} U\right)_{m n}}{\left(\mathcal{E}_{m}-\mathcal{E}_{n}\right)^{2}} .
$$

Here $f_{n} \equiv f_{n}(\mathbf{k})$ and $f_{m} \equiv f_{m}(\mathbf{k})$ are the equilibrium distribution functions for band $n$ and $m$, respectively. The sums in Eq. (84) run over all Wannier states used for an accurate description of the occupied states. Interestingly, this is the standard form of the Berry curvature for a Hamiltonian which depends parametrically on $\mathbf{k}$ and it also shows up as one of the contributions in the KKR and the tight-binding approaches discussed in sections 2.1 and 2.2. Reassuringly, 
computations by all three methods find the contribution from such terms as Eq. (85) dominant and almost exclusively responsible for the spiky features as functions of $\mathbf{k}$. As noted in the introduction, these features originate from band crossings or avoided crossings and have a variety of interesting physical consequences.

For instance, the Berry curvature calculated by Wang et al. [21, 31], shown in Fig. 13, leads directly to a good quantitative account of the intrinsic contribution to the anomalous Hall effect in Fe. The left panel of Fig. 13 shows the decomposition of the Berry curvature in different
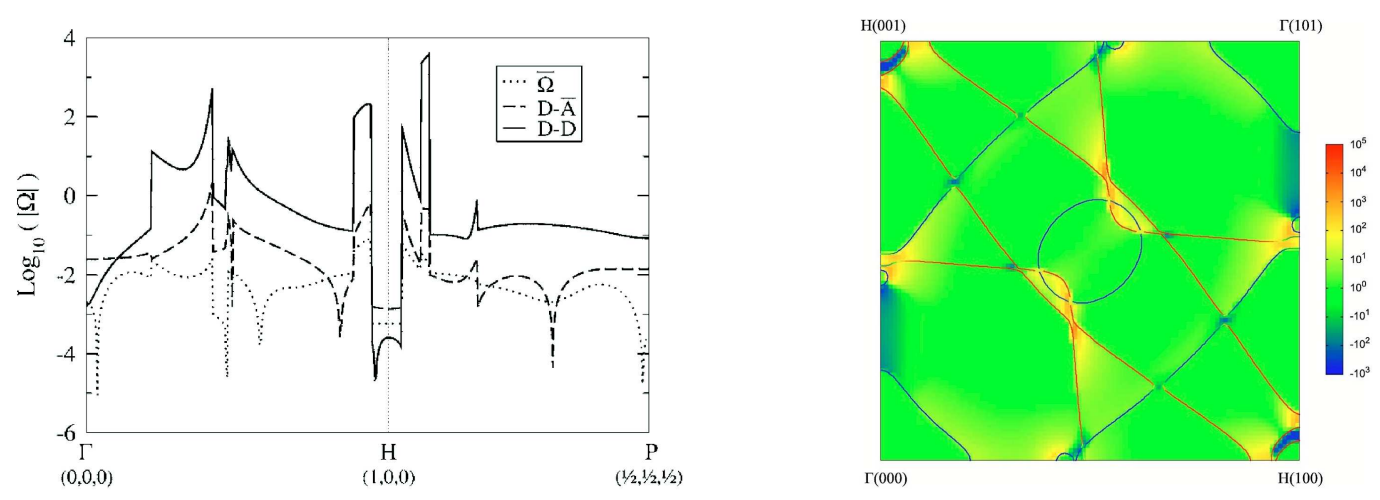

Figure 13: Left: Berry curvature $\Omega^{z}(\mathbf{k})$ of Fe along symmetry lines with a decomposition in three different contributions of Eq. (84) (note the logarithmic scale). Right: Fermi surface in (010) plane (solid lines) and Berry curvature $-\Omega^{z}(\mathbf{k})$ in atomic units (color map) [21].

contributions arising from the expansion of the states into a Wannier basis (see Eq. (84)). Noting the logarithmic scale, it is evident that the dominant contribution stems from the Berry curvature $\boldsymbol{\Omega}^{D D}(\mathbf{k})$ of the $\mathbf{k}$-dependent Hamiltonian $\bar{H}^{H}(\mathbf{k})$ according to Eq. (85). The other terms including matrix elements of the position operator $\mathbf{r}$ are negligible, which is similar to what turned out to be the case within the KKR method. Further, applications of the considered method to the cases of fcc $\mathrm{Ni}$ and hcp $\mathrm{Co}[31,32]$ are equally impressive.

\subsection{Kubo formula}

\subsubsection{Anomalous Hall conductivity and the Berry curvature}

Most conventional approaches to the electronic transport in solids are based on the very general linear response theory of Kubo [33]. Indeed, the first insight into the cause of the anomalous Hall effect by Karplus and Luttinger [34] was gained by deploying the Kubo formula for a simple model Hamiltonian of electrons with spin. In this Section we review briefly the first principles implementation of the Kubo formula in aid of calculating the Berry curvature. The simple observation which makes this possible is that both the semiclassical description and the Kubo formula approach yield the same expression for the intrinsic contribution to the anomalous Hall conductivity (AHC). Hence by comparing the two, a Kubo-like expression for the Berry curvature can be extracted. Here we examine the formal connection between the two formulas for the Berry curvature and demonstrate that they are equivalent as was mentioned already by Wang et al. [21].

The Kubo formula for the AHC in the static limit for disorder-free non-interacting electrons is 
given by [35]

$$
\sigma_{x y}=e^{2} \hbar \int_{B Z} \frac{d^{3} \mathbf{k}}{(2 \pi)^{3}} \sum_{n} \sum_{m \neq n} f_{n}(\mathbf{k}) \frac{\operatorname{Im}\left[\left\langle\Psi_{n \mathbf{k}}|\hat{\mathbf{v}}| \Psi_{m \mathbf{k}}\right\rangle \times\left\langle\Psi_{m \mathbf{k}}|\hat{\mathbf{v}}| \Psi_{n \mathbf{k}}\right\rangle\right]_{z}}{\left(\mathcal{E}_{n_{\mathbf{k}}}-\mathcal{E}_{m \mathbf{k}}\right)^{2}},
$$

whereas in the semiclassical approach it is expressed in terms of the Berry curvature $[14,7,34,41]$

$$
\sigma_{x y}=-\frac{e^{2}}{\hbar} \sum_{n} \int_{B Z} \frac{\mathrm{d}^{3} \mathrm{k}}{(2 \pi)^{3}} f_{n}(\mathbf{k}) \Omega_{n}^{z}(\mathbf{k}) .
$$

Assuming the equivalence of both approaches a comparison of Eqs. (86) and (87) yields in a Kubo-like formula for the Berry curvature. However, the equivalence is not a priori evident and has to be proven which will be done in the following.

The starting point is the Berry curvature written in terms of the periodic part of the Bloch function

$$
\boldsymbol{\Omega}_{n}(\mathbf{k})=i\left\langle\nabla_{\mathbf{k}} u_{n}(\mathbf{k})|\times| \nabla_{\mathbf{k}} u_{n}(\mathbf{k})\right\rangle .
$$

Let us follow the route given by M. V. Berry [3] to rewrite this expression. Introducing the completeness relation with respect to the $N$ present bands, $1=\sum_{m=1}^{N}\left|u_{m}\right\rangle\left\langle u_{m}\right|$, excluding the vanishing term with $m=n$ and using the relation

$$
\left\langle\nabla u_{n}(\mathbf{k}) \mid u_{m}(\mathbf{k})\right\rangle=\frac{\left\langle u_{n}(\mathbf{k})\left|\nabla_{\mathbf{k}} H(\mathbf{k})\right| u_{m}(\mathbf{k})\right\rangle}{\mathcal{E}_{n \mathbf{k}}-\mathcal{E}_{m \mathbf{k}}},
$$

which follows from $\left\langle u_{n}(\mathbf{k})|H(\mathbf{k})| u_{m}(\mathbf{k})\right\rangle=\mathcal{E}_{m \mathbf{k}}\left\langle u_{n}(\mathbf{k}) \mid u_{m}(\mathbf{k})\right\rangle=0$, yields

$$
\boldsymbol{\Omega}_{n}(\mathbf{k})=i \sum_{m \neq n} \frac{\left\langle u_{n}(\mathbf{k})|\nabla H(\mathbf{k})| u_{m}(\mathbf{k})\right\rangle \times\left\langle u_{m}(\mathbf{k})|\nabla H(\mathbf{k})| u_{n}(\mathbf{k})\right\rangle}{\left(\mathcal{E}_{n \mathbf{k}}-\mathcal{E}_{m \mathbf{k}}\right)^{2}} .
$$

If we reformulate it with respect to the Bloch functions

$$
\boldsymbol{\Omega}_{n}(\mathbf{k})=i \sum_{m \neq n} \frac{\left\langle\Psi_{n \mathbf{k}}\left|e^{i \mathbf{k r}} \nabla H(\mathbf{k}) e^{-i \mathbf{k r}}\right| \Psi_{m \mathbf{k}}\right\rangle \times\left\langle\Psi_{m \mathbf{k}}\left|e^{i \mathbf{k r}} \nabla H(\mathbf{k}) e^{-i \mathbf{k r}}\right| \Psi_{n \mathbf{k}}\right\rangle}{\left(\mathcal{E}_{n \mathbf{k}}-\mathcal{E}_{m \mathbf{k}}\right)^{2}}
$$

and use the relations

$$
\begin{gathered}
H(\mathbf{k})=e^{-i \mathbf{k r}} \hat{H} e^{i \mathbf{k r}}, \\
\nabla H(\mathbf{k})=i e^{-i \mathbf{k r}}[\hat{H} \mathbf{r}-\mathbf{r} \hat{H}] e^{i \mathbf{k r}}=\hbar e^{-i \mathbf{k r}} \hat{\mathbf{v}} e^{i \mathbf{k r}},
\end{gathered}
$$

we end up with a Kubo-like formula widely used in the literature [37, 38]

$$
\boldsymbol{\Omega}_{n}(\mathbf{k})=i \hbar^{2} \sum_{m \neq n} \frac{\left\langle\Psi_{n \mathbf{k}}|\hat{\mathbf{v}}| \Psi_{m \mathbf{k}}\right\rangle \times\left\langle\Psi_{m \mathbf{k}}|\hat{\mathbf{v}}| \Psi_{n \mathbf{k}}\right\rangle}{\left(\mathcal{E}_{n \mathbf{k}}-\mathcal{E}_{m \mathbf{k}}\right)^{2}} .
$$

This formula proofs the equivalence of the two approaches for calculating the AHC as given by Eqs. (86) and (87). In prinicple, all occupied and unoccupied states have to be accounted in the sum of Eq. (94). However, in practice only states with energies close to $\mathcal{E}_{n \mathbf{k}}$ play a role. An important feature of this form is that it is expressed in terms of the off-diagonal matrix elements of the velocity operator with respect to the Bloch states. To deal with them a technique was adopted, which served well for computing the optical conductivities [39, 40], where the same matrix elements have been required. 


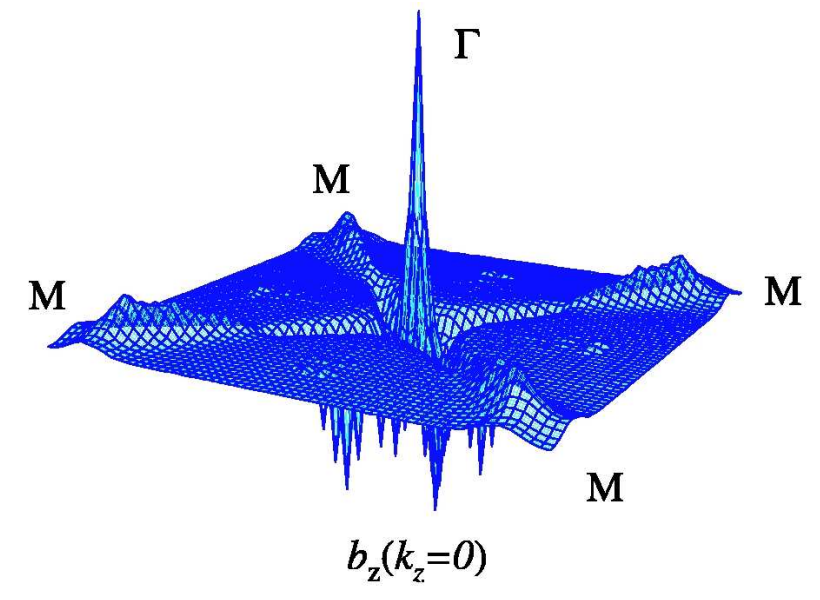

Figure 14: Calculated Berry curvature (in Ref. [36] called flux $\left.b_{z}\right) \Omega^{z}(\mathbf{k})=\sum_{n \in\left\{t_{2 g}\right\}} \Omega_{n}^{z}(\mathbf{k})$ ) distribution in $\mathbf{k}$ space for $t_{2 g}$ bands as a function of $\left(k_{x}, k_{y}\right)$ with $k_{z}=0$ for $\mathrm{SrRuO}_{3}$ with cubic structure [36].

The first ab initio calculation of the Berry curvature was actually performed for $\mathrm{SrRuO}_{3}$ by Fang et al. [36] using Eq. (94) . The authors nicely illustrate the existence of a magnetic monopole in the crystal momentum space, which is shown in Fig. 14. The origin of this sharp structure is the near degeneracy of bands. It acts as a magnetic monopole. A similar effect was found for Fe by Yao et al. [41]. They demonstrate that for $\mathbf{k}$ points near the spin-orbit driven avoided crossings
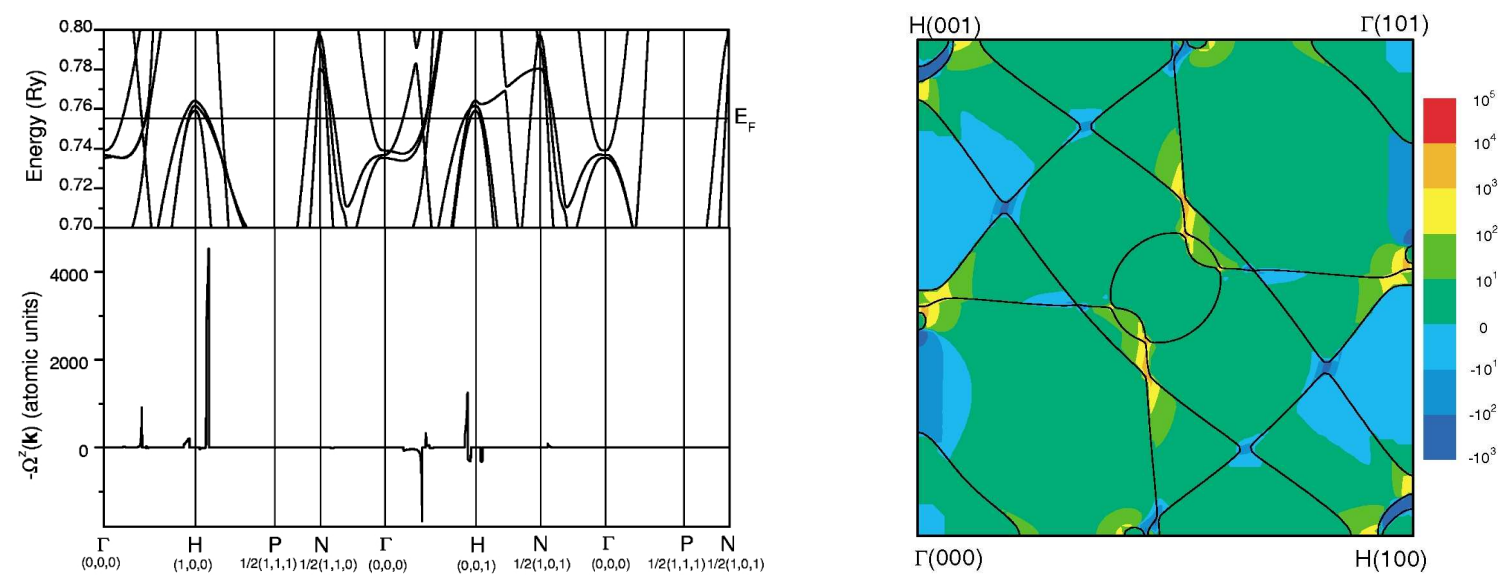

Figure 15: Left: Band structure of bulk Fe near Fermi energy (upper panel) and Berry curvature $\Omega^{z}(\mathbf{k})$ (lower panel) along symmetry lines. Right: Fermi surface in (010) plane (solid lines) and Berry curvature $-\Omega^{z}(\mathbf{k})$ in atomic units (color map) [41].

of the bands the Berry curvature is extremely enhanced as shown in Fig. 15. In addition, the agreement between the right panels of Fig. 13 and Fig. 15 shows impressively the equivalence between the two different methods. 


\subsubsection{Spin Hall conductivity and the so-called spin Berry curvature}

Of course, the fact that the results of the semiclassical transport theory and the quantum mechanical Kubo formula agree exactly, as was shown above, is surprising. In general, it cannot be expected for all transport coefficients. Indeed, as we shall now demonstrate, the situation is quite different for the spin Hall conductivity.

Evidently, the Kubo approach is readily adopted to calculate the spin current response to an external electric field E. Although there is still a controversy about an expression for the spincurrent operator to be taken $[42,43,44,45]$, frequently the following tensor product of the relativistic spin operator $\hat{\beta} \hat{\boldsymbol{\Sigma}}$ and the velocity operator $\hat{\mathbf{v}}$ is used

$$
\hat{\mathbf{J}}^{\mathrm{Spin}}=\hat{\beta} \hat{\boldsymbol{\Sigma}} \otimes \hat{\mathbf{v}} \quad, \quad \hat{\beta} \hat{\boldsymbol{\Sigma}}=\left(\begin{array}{rr}
\hat{\boldsymbol{\sigma}} & 0 \\
0 & -\hat{\boldsymbol{\sigma}}
\end{array}\right) .
$$

Furthermore, the symmetrized version of the tensor product is often used in literature and the spin current response is calculated as the expectation value of the operator $\hat{\mathbf{J}}^{\text {Spin }}$. However, within the Dirac approach we have

$$
\hat{\mathbf{v}}=c \hat{\boldsymbol{\alpha}}=c\left(\begin{array}{cc}
0 & \hat{\boldsymbol{\sigma}} \\
\hat{\boldsymbol{\sigma}} & 0
\end{array}\right) \text { and } \hat{\beta} \hat{\Sigma}_{i} \hat{v}_{j}-\hat{v}_{j} \hat{\beta} \hat{\Sigma}_{i}=0, \text { if } i \neq j .
$$

Similar to the formula for the charge Hall conductivity, one finds [43, 37, 38]

$$
\sigma_{x y}^{z}=\frac{e^{2}}{\hbar} \sum_{\mathbf{k}, n} \sigma_{x y ; n}^{z}(\mathbf{k}) f_{n}(\mathbf{k})
$$

where the $\mathbf{k}$ and band resolved conductivity $\sigma_{y x ; n}^{z}(\mathbf{k})$ in the framework of the Kubo formula (Kubo) is given by

$$
\sigma_{x y ; n}^{z}(\mathbf{k})_{\text {Kubo }}=i \hbar^{2} \sum_{m \neq n} \frac{\left\langle\Psi_{n \mathbf{k}}\left|\hat{\beta} \hat{\Sigma}_{z} \hat{v}_{x}\right| \Psi_{m \mathbf{k}}\right\rangle\left\langle\Psi_{m \mathbf{k}}\left|\hat{v}_{y}\right| \Psi_{n \mathbf{k}}\right\rangle}{\left(\mathcal{E}_{n \mathbf{k}}-\mathcal{E}_{m \mathbf{k}}\right)^{2}}
$$

In the literature, this quantity is sometimes even called spin Berry curvature [38] analogously to the AHE. This notation is misleading and should not be used.

Let us tackle the same problem from the point of view of the semiclassical theory (sc). This suggests that one should take the velocity in Eq. (95) to be the anomalous velocity given by Eq. (11). This argument leads to [5, 42]

$$
\sigma_{x y ; n}^{z}(\mathbf{k})_{\mathrm{sc}}=\operatorname{Tr}\left[\overline{\bar{S}}_{n}^{z}(\mathbf{k}) \overline{\bar{\Omega}}_{n}^{z}(\mathbf{k})\right]
$$

Here $\left(\overline{\bar{S}}_{n}^{z}(\mathbf{k})\right)_{i j}=\left\langle\Psi_{n i \mathbf{k}}\left|\hat{\beta} \hat{\Sigma}_{z}\right| \Psi_{n j \mathbf{k}}\right\rangle$ is the spin matrix for a Kramers pair labeled by $i, j$ and $\overline{\bar{\Omega}}_{n}^{z}(\mathbf{k})$ is the non-Abelian Berry curvature [19] introduced in Section 1.3. The point we wish to make here is that this formula is not equivalent to the Kubo form in Eq. (98). The reason is that the band off-diagonal terms of the spin matrix were neglected in the derivation from the wave packet dynamics of Eq. (99) [5].

Evidently, $\sigma_{x y ; n}^{z}(\mathbf{k})_{\text {Kubo }}$ can not be written as a single band expression, in contrast to the case of the AHC which turned out to be exactly the conventional Berry curvature (see Section 2.4.1). The contributions of Eq. (98) which were neglected in Eq. (99) are of quantum-mechanical origin 
and are not accounted for in the semiclassical derivation. Culcer et al. [42] tackled the problem and identified the neglected contributions, without giving up the wave packet idea, as spin and torque dipole terms. Nevertheless, it was shown [19] that under certain approximations a semiclassical description may result in quantitatively comparable results to the Kubo approach. This will be discussed in the next section.

\section{Intrinsic contribution to the charge and spin conductivity in metals}

Here we discuss applications of the computational methods for the Berry curvature discussed in the previous Section. We will focus on first principles calculations of the anomalous and spin Hall conductivities. The anomalous Nernst conductivity, closely related to it will be also discussed briefly.

The first $a b$ initio studies of the AHE, based on Eq. (87) with $\boldsymbol{\Omega}_{n}(\mathbf{k})$ defined by Eq. (94), were reported in Refs. [36, 46]. As it is well known, the conventional expression for the Hall resistivity

$$
\rho_{x y}=R_{0} B_{z}+4 \pi R_{s} M_{z}
$$

(where $B_{z}$ is the magnetic field in $z$ direction, $R_{0}$ and $R_{s}$ are the normal and the anomalous Hall coefficient, respectively) assumes a monotonic behavior of $\rho_{x y}$ as well as $\sigma_{x y}$ as a function of the magnetization $M$. By means of first principles calculations based on the pseudopotential method (STATE code), Z. Fang et al. [36] have shown that the unconventional nonmonotonic behavior of the AHC measured in $\mathrm{SrRuO}_{3}$ (Fig. 16, left) is induced by the presence of a magnetic monopole (MM) in momentum space (see Fig. 14 and the corresponding discussion in Sec. 2.4.1). The existence of MMs causes the sharp and spiky structure of the AHC as a function of the
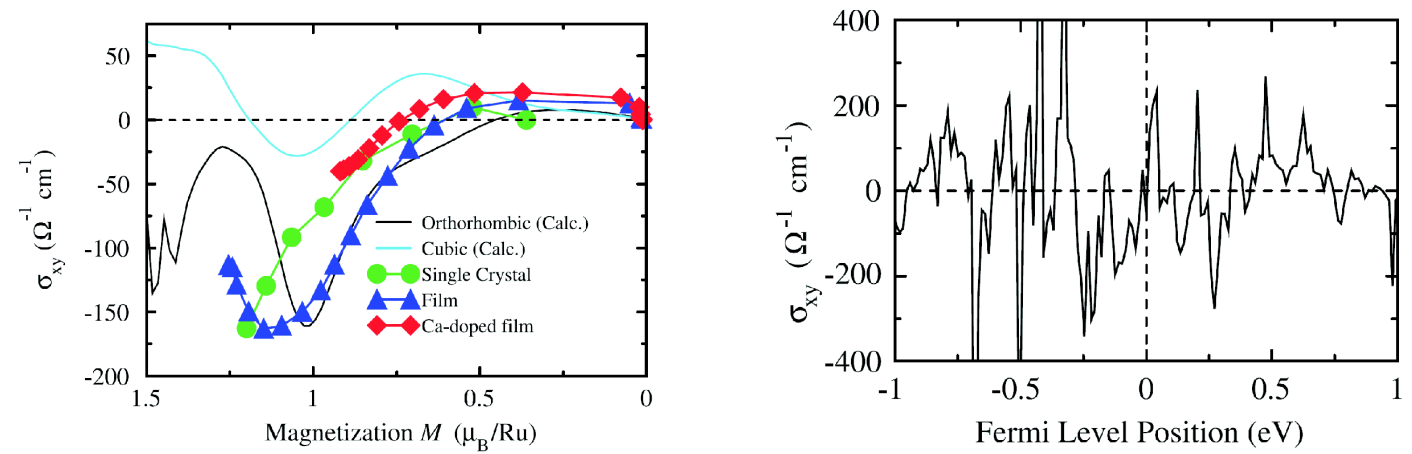

Figure 16: Left: The Hall conductivity $\sigma_{x y}^{z}$ of $\mathrm{SrRuO}_{3}$ as a function of the magnetization along $z$; Right: $\sigma_{x y}^{z}$ as a function of the Fermi-level position for the orthorhombic structure of $\mathrm{SrRuO}_{3}$. [36].

Fermi-level position, shown in the right panel of Fig. 16. At the self-consistently determined Fermi level the calculated value $\sigma_{x y}=-60(\Omega \mathrm{cm})^{-1}$ is comparable with the experimentally observed conductivity of about $-100(\Omega \mathrm{cm})^{-1}$. Obviously, a small change in the Fermi level would cause dramatic changes in the resulting AHC. Indeed, as it was shown in Ref. [46], the 
calculated AHC of ferromagnetic $\mathrm{Gd}_{2} \mathrm{Mo}_{2} \mathrm{O}_{7}$ and $\mathrm{Nd}_{2} \mathrm{Mo}_{2} \mathrm{O}_{7}$ is strongly changed by the choice of the Coulomb repulsion $U$ varied in the used mean-field Hartree-Fock approach.

A year later, results for the intrinsic AHC in ferromagnetic bcc Fe, based on the full-potential linearized augmented plane-wave method (WIEN2K code) were published [41]. The authors used the same Kubo formula approach to describe the transversal transport. In particular, a close agreement between theory, $\sigma_{x y}=751(\Omega \mathrm{cm})^{-1}$, and experiment, $\sigma_{x y}=1032(\Omega \mathrm{cm})^{-1}$, was found that points to the dominance of the intrinsic contribution in Fe. A slow convergence of the calculated value for the $\mathrm{AHC}$ with respect to the number of $\mathbf{k}$ points was reported. The reason for the convergence problems is given by small regions in momentum space around avoided crossings and enhanced spin-orbit coupling. In the behavior of the Berry curvature these points cause strong peaks which is shown in Fig. 15. If both related states of the avoided crossing are occupied their combined contribution to the AHC is negligible since they compensate each other. However, when the Fermi level lies in a spin-orbit induced gap then the occupied state, which acts now as an isolated magnetic monopole, causes a peak in the AHC. Consequently, it was necessary to use millions of $\mathbf{k}$ points in the first Brillouin zone to reach convergence.

To avoid such demanding computational efforts, the Wannier interpolation scheme, discussed in Section 2.3, was suggested and applied for Fe in Ref. [21]. The authors started with the relativistic electronic structure obtained by the pseudopotential method (PWSCF code) at a relatively coarse $\mathbf{k}$ mesh. Using maximally localized Wannier functions, constructed from the obtained Bloch states, all quantities of interest were expressed in the tight-binding like basis and interpolated onto a dense $\mathbf{k}$ mesh. Then this new mesh was used to calculate the AHC. The obtained value of $756(\Omega \mathrm{cm})^{-1}$ is in good agreement with the result of Ref. [41].

The same scheme was used further on to calculate the AHC applying Haldane's formula [16] which means integration not over the entire Fermi sea, as it is required by the Kubo formula, but only over the Fermi surface. The results obtained for Fe, Co, and Ni [31] agreed very well for both procedures and with previous theoretical studies [41]. Moreover, the work done by the group of D. Vanderbilt [21] has stimulated further first-principles calculations as will be shown below.
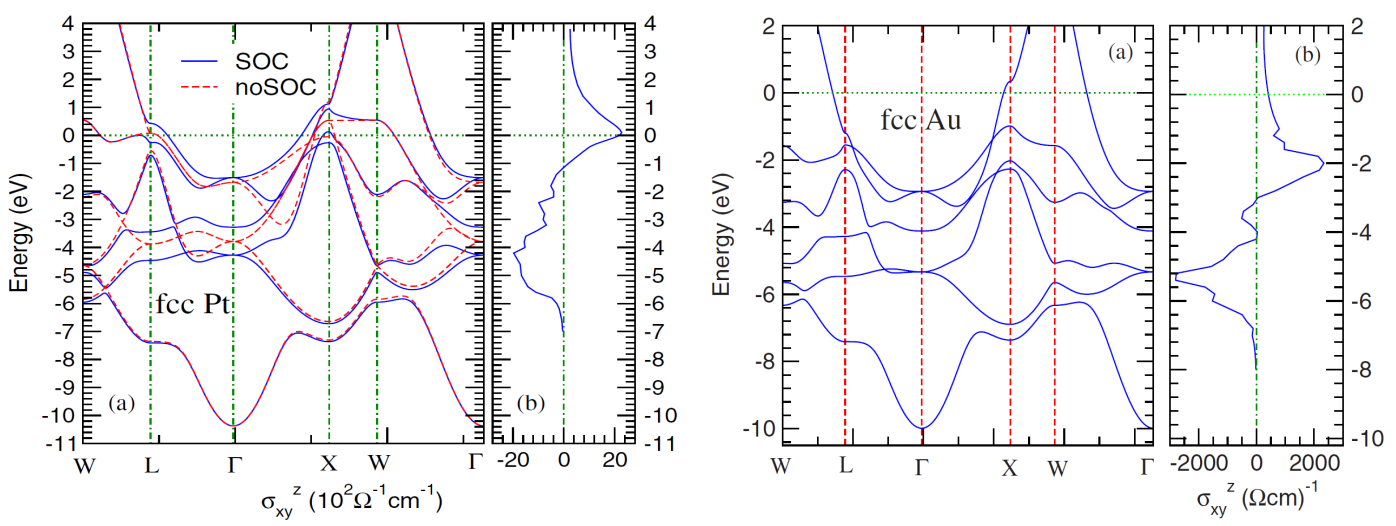

Figure 17: Left: (a) Relativistic band structure and (b) spin Hall conductivity for variable Fermi level of fcc Pt [47]; Right: (a) Relativistic band structure and (b) spin Hall conductivity of fcc $\mathrm{Au}$ [48]. The dashed curves in the left panel represent the scalar-relativistic band structure. The real Fermi level is set as zero of the energy scale. 
The first $a b$ initio calculations of the spin Hall conductivity (SHC) were performed for semiconductors and metals [37, 38]. The description of the electronic structure in Ref. [38] was based on the full-potential linearized augmented plane-wave method (WIEN2K code) and in Ref. [37] on the all-electron linear muffin-tin orbital method, but both rely on the solution of the Kubo formula given by Eq. (98) to describe the SHC. Later, in Ref. [47] the relatively large SHC measured in Pt was explained by the contribution of the spin-orbit split $d$ bands at the high-symmetry points $L$ and $X$ near the Fermi level (Fig. 17, left). In contrast, Au [48] shows similar contributions to the $\mathrm{SHC}$, but at lower energies with respect to the Fermi level $E_{F}$, since the extra electron of Au with respect to Pt causes full occupation of the $d$ bands (Fig. 17, right). The spin-orbit coupling is the origin of the AHE and the SHE. In particular, the avoided crossings significantly increase these effects. In addition, the spin-orbit coupling links the spin degree of freedom to the crystal lattice, which is the source of the anisotropy with respect to the chosen quantization axis, this means $\sigma_{y z}^{x}$ and $\sigma_{x y}^{z}$ may differ. This was demonstrated first for the AHE in hcp Co [32] and later for the SHE in several nonmagnetic hcp metals [49] (Fig. 18). Here, the pseudopotential method (PWSCF code) and the full-potential linearized augmented-plane-wave method (FLEUR code) were used for the AHE and SHE, respectively. To reduce the computational efforts, the Berry curvature calculations were based on the Wannier interpolation scheme according to Ref. [21]. These methods were applied to several situations in ordered alloys [50] where the anisotropy of the AHC [51] was dicussed as well and the role of spin-conserving and non-spin-conserving parts to the SOC were discussed in detail [52].

Finally, an approach for calculating the SHC based on the KKR method was proposed (Ref. [19], Section 2.1). For the description of the transversal spin transport the authors applied an approach different from the widely used Kubo formula. Their calculations are based on the expression for the SHC in the non-Abelian case suggested in Ref. [5]. This expression was simplified further by the approximation that the expectation value of the $\hat{\beta} \hat{\Sigma}_{z}$ operator is assumed to be +1 and -1 for the two spin degenerate states, arising in nonmagnetic crystals with inversion symmetry. Thus, the semiclassical treatment was reduced to the two current model with the final expression for the SHC in terms of the usual formula for the AHC at zero temperature

$$
\sigma_{x y}^{z}=\frac{e^{2}}{\hbar(2 \pi)^{3}} \int^{E_{F}} d \mathcal{E} \Omega^{z}(\mathcal{E}),
$$
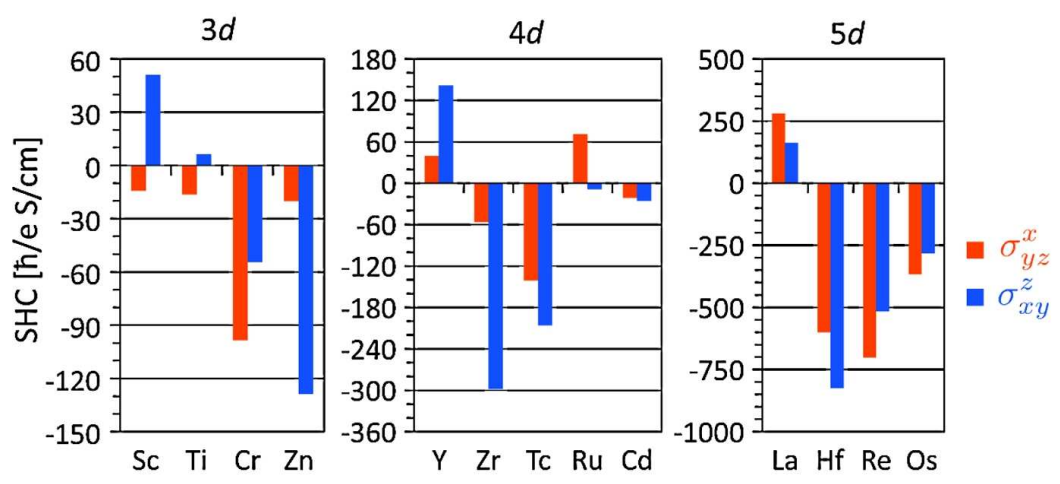

Figure 18: Spin Hall conductivities $\sigma_{y z}^{x}$ and $\sigma_{x y}^{z}$ for several hcp nonmagnetic metals and for antiferromagnetic Cr [49]. 

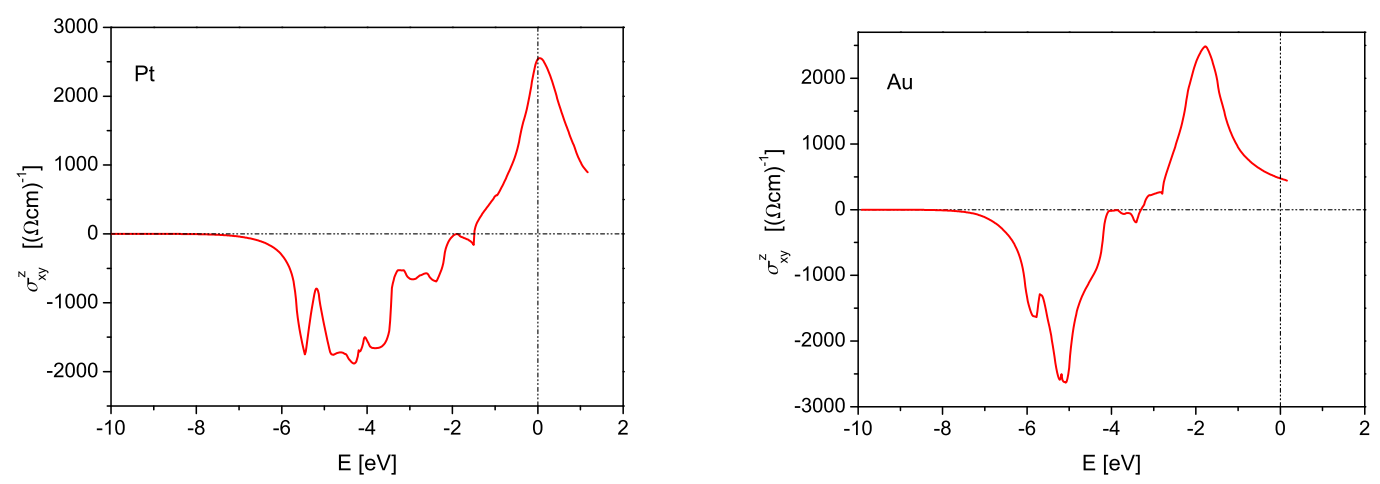

Figure 19: The spin Hall conductivity for fcc Pt (left) and Au (right) [19].

where $\Omega^{z}(\mathcal{E})$ is defined by Eq. (65). The spin Hall conductivities $\sigma_{x y}^{z}(\mathcal{E})$ considered for a variable Fermi level, and calculated for $\mathrm{Pt}$ and $\mathrm{Au}$ in such an approach (Fig. 19), are quantitatively in good agreement to the ones obtained by the Kubo-like formula (see Fig. 17).

Related to the already discussed calculation of the intrinsic spin and anomalous Hall conductivities is the intrinsic mechanism of the anomalous Nernst effect. It is driven by an applied thermal gradient instead of the applied electric field. Both driving forces are of physically different nature. The electric field is a mechanical force in contrast to the statistical force of a temperature gradient. Nevertheless, as was shown by Xiao et al. [53], the final expression for the anomalous Nernst conductivity $\alpha_{x y}$ at low temperatures is simply given by the Mott relation

$$
\alpha_{x y}=\frac{\pi^{2}}{3} \frac{k_{B}^{2} T}{e} \sigma_{x y}^{\prime}\left(E_{F}\right) .
$$

Here $\sigma_{x y}^{\prime}\left(E_{F}\right)$ is the first energy derivative at $E_{F}$ of the intrinsic anomalous Hall conductivity, defined in terms of the Berry curvature in Eq. (87). All already discussed methods to calculate the Berry curvature can be applied to compute the anomalous Nernst conductivity as well. In Fig. 20 a comparison between experimental results and first principle calculations for the alloy $\mathrm{CuCr}_{2} \mathrm{Se}_{4-x} \mathrm{Br}_{x}$ are shown. The concentration dependence is described by using a rigid band model. The agreement is relatively good despite the fact that the experiment can not resolve the peak-valley structure around $x=0.2$.

\section{De Haas-van Alphen oscillation and the Berry phases}

Other phenomena related to the topic of this review are various quantum oscillations. For their proper description typically the Berry phase accumulated along a closed orbits in parameter space is needed which can be expressed via the Berry curvature of the system. For instance, in case of the de Haas-van Alphen effect, the semiclassical formula for the quantized energies of the Landau levels includes a term involving the Berry phase of the corresponding orbits in k space. This was pointed out by Mikitik and Sharlai $[54,55]$ who have discussed a number of interesting experiments in these terms $[56,57]$. They argue that the quasiparticles which move along closed orbits in response to an external magnetic field $\mathbf{B}$ are wave packets, in the sense of the semiclassical theory, and therefore their semiclassical Eqs. (8) and (9) are subject 


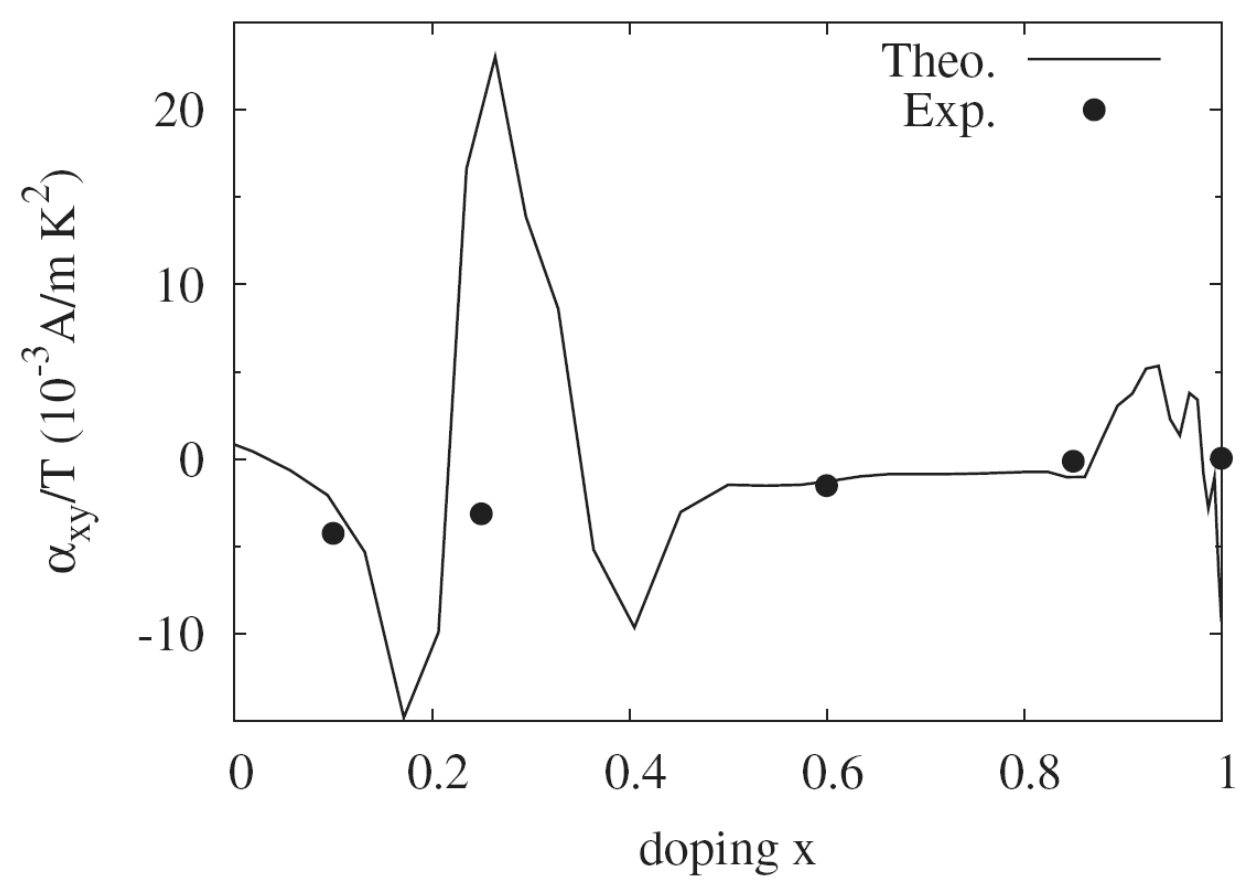

Figure 20: The intrinsic anomalous Nernst conductivity (divided by the temperature $\mathrm{T}$ ) of the $\mathrm{CuCr}_{2} \mathrm{Se}_{4-x} \mathrm{Br}_{x}$ alloy as a function of the Br content x [53].

to corrections arising from Berry phases and curvatures. In short, a closed orbit is defined by the requirement that the energy of the wave packet $\mathcal{E}_{n \mathbf{k}}-\mathbf{M}(\mathbf{k}) \mathbf{B}$ is equal to $\mathrm{E}$ for all $\mathbf{k}$ along the orbit. Here $\mathbf{M}(\mathbf{k})$ is the spin plus the orbital magnetic moment of the electron along the path. Then the Bohr-Sommerfeld quantization condition for $E=E_{\nu}$ to be a Landau level is $[55,58,1]$

$$
S\left(E_{\nu}, k_{z}\right)=2 \pi \hbar e B_{z}\left(\nu+\frac{1}{2}-\frac{\gamma_{B}}{2 \pi}\right)
$$

where $S\left(E_{\nu}, k_{z}\right)$ is the area enclosed by the orbit in $\mathbf{k}$ space. The Berry phase accumulated around the orbit labeled by $C$ is given by

$$
\gamma_{B}=\oint_{C} \mathrm{~d} \mathbf{k} \cdot \mathbf{A}_{n}(\mathbf{k})=\iint_{S_{c}} d \mathbf{S} \cdot \Omega_{n}(\mathbf{k}),
$$

where $S_{c}$ is any surface in $\mathbf{k}$ space whose boundary is $C$. Clearly, the presence of the orbital magnetization in the theory indicates that the change of the band energy due to spin and orbital motion have been taken into account. This important fact is more evident if Eq. (103) is recast as

$$
S_{0}\left(\mathcal{E}_{n \mathbf{k}}, k_{z}\right)=2 \pi \hbar e B_{z}\left(\nu+\frac{1}{2}-\frac{\gamma_{B}}{2 \pi}-\frac{1}{2 \pi} \oint \frac{M_{z}(k) d_{k}}{\left|\partial \mathcal{E}_{n \mathbf{k}} / \partial k_{\perp}\right|}\right)
$$

where $S_{0}\left(\mathcal{E}_{n \mathbf{k}}, k_{z}\right)$ is the area enclosed by an orbit on an isosurface of the actual band structure. From the point of view of analyzing the high quality data available from de Haas-van Alphen measurements, the physical content of the above formula is of considerable general interest. However, to calculate the loop integral in Eq. (105) for the general case with spin-orbit coupling is a demanding task which has been, so far, only partially implemented [59]. 

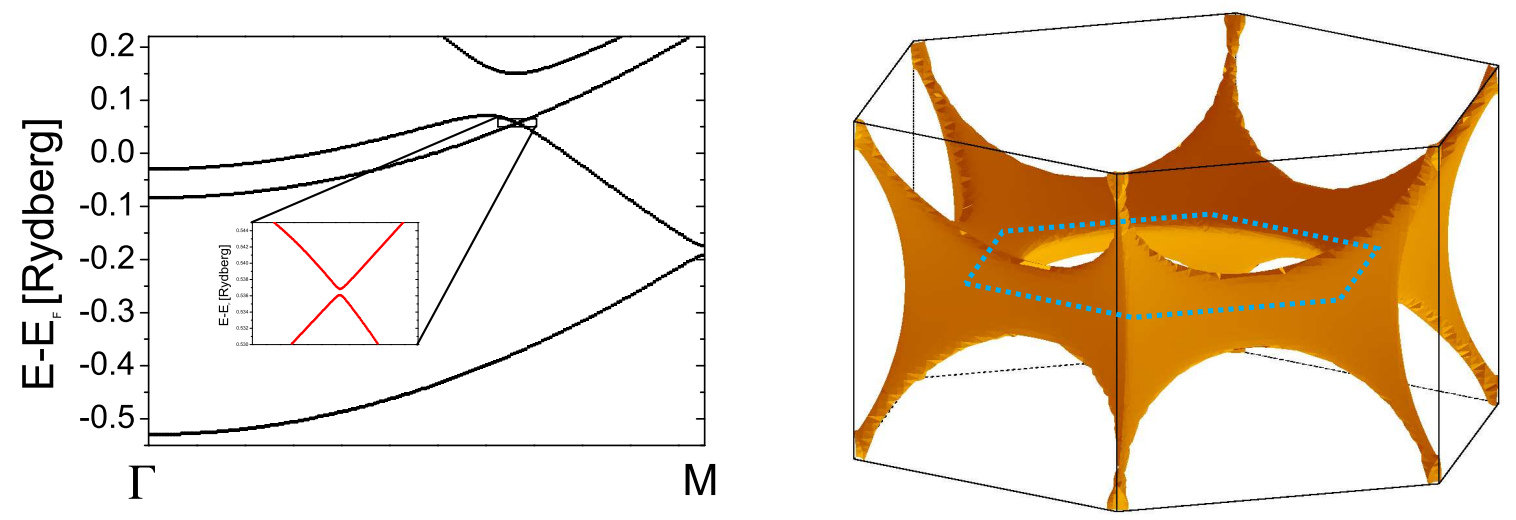

Figure 21: Left panel: Band structure of $\mathrm{Mg}$ along the $\Gamma M$ direction. The accidental degeneracy of two bands is part of a line of degeneracy in the $k_{z}=0$ plane. The inset shows the lifting of the degeneracy due to spin-orbit coupling. Right panel: The full line of degeneracy which is enclosed by the Fermi surface.

In concluding this brief summary of the problem it should be stressed that the formulas above were derived by L. Roth already in 1966 [60]. The need to revisit these issues has arisen only recently in view of the new light cast on them by advances in describing wave packets driven by external fields and the associated Berry phases.

To highlight the significance of the Berry phase and curvature in the present context we shall now consider the simple case where the spin, and therefore the spin-orbit coupling, is altogether neglected and it is assumed that the system is time-reversal and inversion symmetric. In this case one might think that the Berry phase would vanish since the Berry curvature is zero over the full Brillouin zone. However, as was shown by G.P. Mikitik and Yu.V. Sharlai $[54,55]$ this is not true if the semiclassical orbit enclose a line of degeneracy. As was shown in Ref. $[56,57]$ for several materials such lines exist in absence of spin-orbit coupling and have a significant effect on the observable de Haas-van Alphen frequencies.

Here we will focus on two hexagonal systems, $\mathrm{Mg}$ and $\mathrm{Tl}$, and discuss the results for $\gamma_{B}$, given by Eq. (104), obtained with the KKR method [22, 19]. In Fig. 21 the bandstructure of Mg along the $\Gamma M$ direction clearly shows an accidental degeneracy which is actually part of a full line in the $k_{z}=0$ plane as shown on the right side of Fig. 21. Furthermore, this line is enclosed by one sheet of the Fermi surface which allows to study the effect. If the magnetic field is applied in different direction as shown in Fig. 22 orbits either enclose the line of degeneracy, as in the case shown in red, or they do not. We have evaluated the integrals over a selection of those loops numerically and found $\gamma_{B}=\pi$ for all orbits which enclose the line and $\gamma_{B}=0$ for all those which do not. The important fact is that the final integral is not influenced by the actual form of the loop. The effect is topological since only the fact whether the line penetrates the orbit or not is relevant. This result dramatically affects the analysis of de Haas-van Alphen experiments since the quantization condition of Eq. (103) will be either

$$
S\left(E, k_{z}\right)=2 \pi \hbar e B_{z} \nu
$$


a)

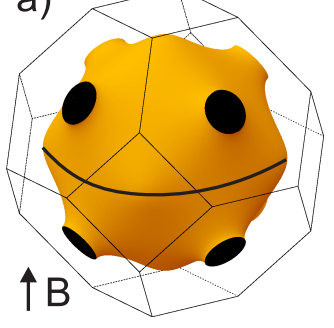

b)

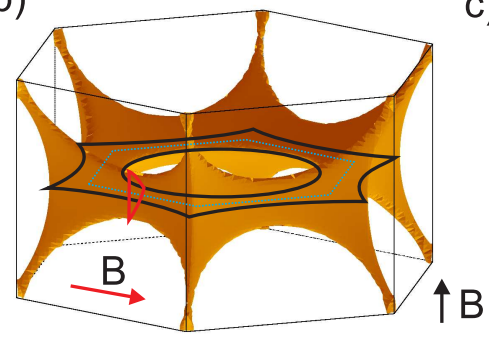

c)

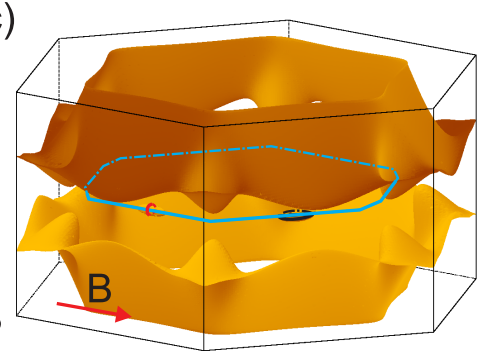

Figure 22: Fermi surfaces for $\mathrm{Cu}(\mathrm{a})$ and $\mathrm{Mg}$ (b) with extremal orbits for different directions of the applied magnetic field. For Tl (c) a constant energy surface slightly above the Fermi energy $\left(E_{F}+0.02\right.$ Rydberg $)$ was chosen to create extremal orbits enclose a line of contact points. The orbits enclosing a line of degeneracy are shown in red others in black. The lines of degeneracy are shown as blue dashed lines.

or

$$
S\left(E, k_{z}\right)=2 \pi \hbar e B_{z}\left(\nu+\frac{1}{2}\right)
$$

depending whether the orbit encloses a line of degeneracies or not, respectively.

Clearly, what happens to such lines of degeneracies in the presence of spin and spin-orbit coupling is an interesting and fundamental question since in that case the bands are formed from states that are Krammer doublets and two such doublets are infinitely unlikely to be degenerate. In the limit of small spin-orbit coupling this question was investigated by Mikitik and Sharlai [61]. They find that the Berry phase contribution becomes zero but for nonmagnetic inversion symmetric systems there is a contribution to the area enclosed by a Landau orbit arising from the magnetization part of Eq. (105). However the strong coupling limit remains an open problem. 


\section{Conclusion}

In the presence of a periodic crystal potential the free electron spectrum $\mathcal{E}_{\mathbf{k}}=\frac{\hbar^{2} k^{2}}{2 m}$ breaks up into bands with complex dispersion relation $\mathcal{E}_{n \mathbf{k}}$. It turns out that the adiabatic dynamics of wave packets, constructed by superposition of all $\mathbf{k}$ states but a limited number of bands $n$, features a Berry connection $\mathbf{A}_{n}(\mathbf{k})$ and a corresponding curvature $\boldsymbol{\Omega}_{n}(\mathbf{k})$ which account for the influence of the neglected bands [6, 7]. Moreover, these geometrical properties of the band structure play an essential role in describing a number of important physical phenomena such as spin and charge transport, electric polarization and orbital magnetization [1]. What makes these remarkable developments particularly interesting from the point of view of first-principles electronic structure calculations is the fact that the curvature $\boldsymbol{\Omega}_{n}(\mathbf{k})$ is a very rapidly varying function of $\mathbf{k}$ and it depends on intricate details of the band structure. Consequently, devising methods for computing $\boldsymbol{\Omega}_{n}(\mathbf{k})$ have recently become a very active field of research. In this paper we sketched three approaches to the problem: one is based on Multiple Scattering Theory (KKR, similarly LMTO), another one on the Wannier function representation of first-principles bands and the third one on direct evaluation of a Kubo like formula. We pointed out the differences and highlighted the similarities in the way the derivatives with respect to the wave vector $\mathbf{k}$ enter the problem. We stressed the importance of level crossings which give rise to curvatures $\boldsymbol{\Omega}_{n}(\mathbf{k})$ whose $\mathbf{k}$ dependence is that of the Dirac monopole and the more exotic avoided crossings of Kramers degenerate bands whose curvature is a non-Abelian gauge field. As illustrations of results by the various methods, we presented calculations of the intrinsic contribution to the anomalous and spin Hall conductivity, the Berry curvature for a variety of systems as well as the influence of the Berry phase on the de Haas-van Alphen oszillations.

Hopefully, it has been demonstrated that more work is needed to make quantitative contact with experiments on more complex materials and transport phenomena other then those we had occasion to mention. From the computational point of view the spatial and temporal like curvatures, will represent new challenges. 


\section{References}

[1] D. Xiao, M.-C. Chang, and Q. Niu, Berry phase effects on electronic properties, Rev. Mod. Phys. 82, 1959 (2010).

[2] M. P. Marder, Condensed Matter Physics, Wiley, New York (2000).

[3] M. V. Berry, Quantal phase factors accompanying adiabatic changes, Proc. Roy. Soc. A (London) 392, 45 (1984).

[4] D. Culcer, Y. Yao and Q. Niu, Coherent wave-packet evolution in coupled bands, Phys. Rev. B 72, 085110 (2005).

[5] R. Shindou and K.-I. Imura, Noncommutative geometry and non-Abelian Berry phase in the wave-packet dynamics of Bloch electrons, Nuclear Phys. B 720, 399-435 (2005).

[6] F. Wilczek and A. Zee, Appearance of Gauge Structure in Simple Dynamical Systems, Phys. Rev. Lett. 52, 2111 (1984).

[7] G. Sundaram and Q. Niu, Wave-packet dynamics in slowly perturbed crystals: Gradient corrections and Berry-phase effects, Phys. Rev. B 59, 14915 (1999).

[8] H. A. Kramers, General Theory of Paramagnetic Rotation in Crystals, Proc. R. Acad. Sci. Amsterdam 33, 959 (1930).

[9] R. J. Elliott, Theory of the Effect of Spin-Orbit Coupling on Magnetic Resonance in Some Semiconductors, Phys. Rev. 96, 266 (1954).

[10] M. V. Berry and M. Wilkinson, Diabolical Points in the Spectra of Triangles, Proc. Roy. Soc. (London) 392, 15 (1984).

[11] F. Pientka, Geometrical Concepts in the Band Theory of Solids, Diploma thesis, University Halle-Wittenberg (2010).

[12] K. M. D. Hals, A. K. Nguyen, X. Waintal, and A. Brataas, Effective Magnetic Monopoles and Universal Conductance Fluctuations, Phys. Rev. Lett. 105, 207204 (2010).

[13] P. A. M. Dirac, The Theory of Magnetic Poles, Phys. Rev. 74, 817 (1948).

[14] D. J. Thouless, M. Kohmoto, M. P. Nightingale, and M. den Nijs, Quantized Hall Conductance in a Two-Dimensional Periodic Potential, Phys. Rev. Lett. 49, 405 (1982).

[15] B. Simon, Holonomy, the Quantum Adiabatic Theorem, and Berry's Phase, Phys. Rev. Lett. 51, 2167 (1983).

[16] F. D. M. Haldane, Berry Curvature on the Fermi Surface: Anomalous Hall Effect as a Topological Fermi-Liquid Property, Phys. Rev. Lett. 93, 206602 (2004).

[17] J. von Neumann and E. Wigner, Über das Verhalten von Eigenwerten bei adiabatischen Prozessen, Phys. Z. 30, 467 (1929). 
[18] A. Bohm, A. Mostafazadeh, H. Koizumi, Q. Niu, and J. Zwanziger, The Geometric Phase in Quantum Systems, Springer-Verlag, Berlin (2003).

[19] M. Gradhand, D.V. Fedorov, F. Pientka, P. Zahn, I. Mertig, and B.L. Györffy, On calculating the Berry curvature of Bloch electrons using the KKR method, Phys. Rev. B, 84, 075113 (2011).

[20] J. Fabian and S. Das Sarma, Spin Relaxation of Conduction Electrons in Polyvalent Metals: Theory and a Realistic Calculation, Phys. Rev. Lett. 81, 5624 (1998).

[21] X. Wang, J. R. Yates, I. Souza, and D. Vanderbilt, Ab initio calculation of the anomalous Hall conductivity by Wannier interpolation, Phys. Rev. B 74, 195118 (2006).

[22] M. Gradhand, M. Czerner, D.V. Fedorov, P. Zahn, B. Yu. Yavorsky, L. Szunyogh, and I. Mertig, Spin polarization on Fermi surfaces of metals by the KKR method, Phys. Rev. B 80, 224413 (2009).

[23] F. Pientka, M. Gradhand, D.V. Fedorov, I. Mertig, and B.L. Györffy, On a choice of gauge for degenerate Bloch states in nonmagnetic crystals with inversion symmetry, to be submitted.

[24] R. Zeller, P. H. Dederichs, B. Újfalussy, L. Szunyogh, P. Weinberger, Theory and convergence properties of the screened Korringa-Kohn-Rostoker method, Phys. Rev. B 52, 8807 (1995).

[25] L. Szunyogh, B. Újfalussy, P. Weinberger, and J. Kollr Self-consistent localized KKR scheme for surfaces and interfaces, Phys. Rev. B 49, 2721 (1994).

[26] P. Strange, Relativistic Quantum Mechanics with applications in condensed Matter and atomic physics, (Cambridge University Press, 1998).

[27] J. C. Slater and G. F. Koster, Simplified LCAO Method for the Periodic Potential Problem, Phys. Rev. 94, 1498 (1954).

[28] M. D. Jones and R. C. Albers, Spin-orbit coupling in an f-electron tight-binding model: Electronic properties of Th, U, and Pu, Phys. Rev. B 79, 045107 (2009).

[29] N. Marzari, I. Souza, and D. Vanderbilt, An Introduction to Maximally-Localized Wannier Functions, Psi-k Newsletter 57, Scientific Highlight of the month (2003).

[30] N. Marzari and D. Vanderbilt, Maximally localized generalized Wannier functions for composite energy bands, Phys. Rev. B 56, 12847 (1997).

[31] X. Wang, D. Vanderbilt, J. R. Yates, and I. Souza, Fermi-surface calculation of the anomalous Hall conductivity, Phys. Rev. B 76, 195109 (2007).

[32] E. Roman, Y. Mokrousov, and I. Souza, Orientation Dependence of the Intrinsic Anomalous Hall Effect in hcp Cobalt, Phys. Rev. Lett. 103, 097203 (2009).

[33] R. Kubo, Statistical-Mechanical Theory of Irreversible Processes, J. Phys. Soc. Japan 12, 570 (1957). 
[34] R. Karplus and J. M. Luttinger, Hall Effect in Ferromagnetics, Phys. Rev. 95, 1154 (1954).

[35] J. Sinova, T. Jungwirth, J. Kucera, and A. H. MacDonald, Infrared magneto-optical properties of (III,Mn)V ferromagetic semiconductors, Phys. Rev. B 67, 235203 (2003).

[36] Z. Fang, N. Nagaosa, K. S. Takahashi, A. Asamitsu, R. Mathieu, T. Ogasawara, H. Yamada, M. Kawasaki, Y. Tokura, K. Terakura, The Anomalous Hall Effect and Magnetic Monopoles in Momentum Space, Science 302, 92 (2003).

[37] G. Y. Guo, Y. Yao, and Q. Niu, Ab initio Calculation of the Intrinsic Spin Hall Effect in Semiconductors, Phys. Rev. Lett. 94, 226601 (2005).

[38] Y. Yao and Z. Fang, Sign Changes of Intrinsic Spin Hall Effect in Semiconductors and Simple Metals: First-Principles Calculations, Phys. Rev. Lett. 95, 156601 (2005).

[39] C. S. Wang and J. Callaway, Band structure of nickel: Spin-orbit coupling, the Fermi surface, and the optical conductivity, Phys. Rev. B 9, 4897 (1974).

[40] G. Y. Guo and H. Ebert, Band-theoretical investigation of the magneto-optical Kerr effect in Fe and Co multilayers, Phys. Rev. B 51, 12633 (1995).

[41] Y. Yao, L. Kleinman, A.H. MacDonald, J. Sinova, T. Jungwirth, D. Wang, E. Wang, and Q. Niu, First Principles Calculation of Anomalous Hall Conductivity in Ferromagnetic bcc Fe, Phys. Rev. Lett. 92, 037204 (2004).

[42] D. Culcer, J. Sinova, N. A. Sinitsyn, T. Jungwirth, A. H. MacDonald, and Q. Niu, Semiclassical Spin Transport in Spin-Orbit-Coupled Bands, Phys. Rev. Lett. 93, 046602 (2004).

[43] J. Sinova. D. Culcer, Q. Niu, N. A. Sinitsyn, T. Jungwirth, and A. H. MacDonald, Universal Intrinsic Spin Hall Effect, Phys. Rev. Lett. 92, 126603 (2004).

[44] A. Vernes, B. L. Györffy, and P. Weinberger, Spin currents, spin-transfer torque, and spinHall effects in relativistic quantum mechanics, Phys. Rev. B 76, 012408 (2007).

[45] S. Lowitzer, M. Gradhand, D. Ködderitzsch, D.V. Fedorov, I. Mertig, H. Ebert, Extrinsic and Intrinsic Contributions to the Spin Hall Effect of Alloys, Phys. Rev. Lett. 106, 056601 (2011).

[46] I. V. Solovyev, Effects of crystal structure and on-site Coulomb interactions on the electronic and magnetic structure of $A_{2} \mathrm{Mo}_{2} \mathrm{O}_{7}(A=Y, G d$, and $N d)$ pyrochlores, Phys. Rev. B 67, $174406(2003)$

[47] G.Y. Guo, S. Murakami, T.-W. Chen, and N. Nagaosa, Intrinsic Spin Hall Effect in Platinum: First-Principles Calculations, Phys. Rev. Lett. 100, 096401 (2008).

[48] G. Y. Guo, Ab initio calculation of intrinsic spin Hall conductivity of Pd and Au, J. Appl. Phys. 105, 07C701 (2009).

[49] F. Freimuth, S. Blügel, and Y. Mokrousov, Anisotropic Spin Hall Effect from First Principles, Phys. Rev. Lett. 105, 246602 (2010). 
[50] K. M. Seemann, Y. Mokrousov, A. Aziz, J. Miguel, F. Kronast, W. Kuch, M. G. Blamire, A. T. Hindmarch, B. J. Hickey, I. Souza, and C. H. Marrows, Spin-Orbit Strength Driven Crossover between Intrinsic and Extrinsic Mechanisms of the Anomalous Hall Effect in the Epitaxial L1 $1_{0}$-Ordered Ferromagnets FePd and FePt, Phys. Rev. Lett. 104, 076402 (2010).

[51] H. Zhang, S. Blügel, and Y. Mokrousov, Anisotropic intrinsic anomalous Hall effect in ordered 3dPt alloys, Phys. Rev. B. 84, 024401 (2011).

[52] H. Zhang, F. Freimuth, S. Blügel, and Y. Mokrousov, Role of Spin-Flip Transitions in the Anomalous Hall Effect of FePt Alloy, Phys. Rev. Lett. 106, 117202 (2011).

[53] D. Xiao, Y. Yao, Z. Fang, and Q. Niu, Berry-Phase Effect in Anomalous Thermoelectric Transport, Phys. Rev. Lett. 97, 026603 (2006).

[54] G. P. Mikitik and Yu. V. Sharlai, Semiclassical energy levels of electrons in metals with band degeneracy lines, J. Exp. Theor. Phys. 87, 747 (1998).

[55] G. P. Mikitik and Yu. V. Sharlai, Manifestation of Berry's Phase in Metal Physics, Phys. Rev. Lett. 82, 2147 (1999).

[56] G. P. Mikitik and Yu. V. Sharlai, Berry phase and de Haas-van Alphen effect in LaRhIn, Phys. Rev. Lett. 93, 106403 (2004).

[57] G. P. Mikitik and Yu. V. Sharlai, The phase of the Haas-van Alphen oszillations, the Berry phase, and band-contact lines in metals, Low Temp. Phys. 33, 439 (2007).

[58] P. Gosselin, H. Boumrar, and H. Mohrbach, Semiclassical quantization of electrons in magnetic fields: The generalized Peierls substitution, Europhys. Lett. 84, 50002 (2010).

[59] See for example Ref. [62] and citations (9-30) in Ref. [61].

[60] L. M. Roth, Semiclassical theory of magnetic energy levels and magnetic susceptibility of Bloch Electrons, Phys. Rev. B 145, 434 (1966).

[61] G. P. Mikitik and Yu. V. Sharlai, g factor of conduction electrons in the de Haas-van Alphen effect, Phys. Rev. B 65, 184426 (2002).

[62] O. Erikson, H. Ohlsen, and J. L. Calais, Conduction-electron Zeeman splitting in the noble metals Phys. Rev. B 40, 59615966 (1989). 